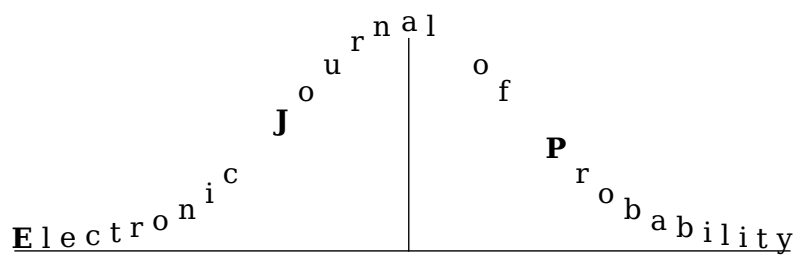

Electron. J. Probab. 26 (2021), article no. 122, 1-31.

ISSN: 1083-6489 https://doi.org/10.1214/21-EJP691

\title{
On the asymptotic of the maximal weighted increment of a random walk with regularly varying jumps: the boundary case
}

\author{
Alfredas Račkauskas* $\quad$ Charles Suquet ${ }^{\dagger}$
}

\begin{abstract}
Let $\left(X_{i}\right)_{i \geq 1}$ be i.i.d. random variables with $\mathrm{E} X_{1}=0$, regularly varying with exponent $a>2$ and $t^{a} P\left(\left|X_{1}\right|>t\right) \sim L(t)$ slowly varying as $t \rightarrow \infty$. We give the limit distribution of $T_{n}(\gamma)=\max _{0 \leq j<k \leq n}\left|X_{j+1}+\cdots+X_{k}\right|(k-j)^{-\gamma}$ in the threshold case $\gamma_{a}:=1 / 2-1 / a$ which separates the Brownian phase corresponding to $0 \leq \gamma<\gamma_{a}$ where the limit of $T_{n}(\gamma)$ is $\sigma T(\gamma)$, with $\sigma^{2}=\mathrm{E} X_{1}^{2}, T(\gamma)$ is the $\gamma$-Hölder norm of a standard Brownian motion and the Fréchet phase corresponding to $\gamma_{a}<\gamma<1$ where the limit of $T_{n}(\gamma)$ is $Y_{a}$ with Fréchet distribution $P\left(Y_{a} \leq x\right)=\exp \left(-x^{-a}\right), x>0$. We prove that $c_{n}^{-1}\left(T_{n}\left(\gamma_{a}\right)-\mu_{n}\right)$, converges in distribution to some random variable $Z$ if and only if $L$ has a limit $\tau^{a} \in[0, \infty]$ at infinity. In such case, there are $A>0, B \in \mathbb{R}$ such that $Z=A V_{a, \sigma, \tau}+B$ in distribution, where for $0<\tau<\infty, V_{a, \sigma, \tau}:=\max \left(\sigma T\left(\gamma_{a}\right), \tau Y_{a}\right)$ with $T\left(\gamma_{a}\right)$ and $Y_{a}$ independent and $V_{a, \sigma, 0}:=\sigma T\left(\gamma_{a}\right), V_{a, \sigma, \infty}:=Y_{a}$. When $\tau<\infty$, a possible choice for the normalization is $c_{n}=n^{-1 / a}$ and $\mu_{n}=0$, with $Z=V_{a, \sigma, \tau}$. We also build an example where $L$ has no limit at infinity and $\left(T_{n}(\gamma)\right)_{n \geq 1}$ has for each $\tau \in[0, \infty]$ a subsequence converging after normalization to $V_{a, \sigma, \tau}$.
\end{abstract}

Keywords: random walk; maximal increment; regularly varying random variables. MSC2020 subject classifications: $60 \mathrm{G} 50 ; 60 \mathrm{G} 70$.

Submitted to EJP on March 30, 2021, final version accepted on August 25, 2021.

\section{Introduction}

Consider a sequence of independent identically distributed (i.i.d.) zero mean random variables $\left(X_{k}, k \geq 1\right)$ and the associated partial sums

$$
S_{0}=0, \quad S_{n}=X_{1}+\cdots+X_{n}, \quad n \geq 1 .
$$

*Institute of Applied Mathematics, Faculty of Mathematics and Informatics, Vilnius University, Naugarduko 24, LT-03225, Vilnius, Lithuania. E-mail: al fredas. rackauskas@mif.vu. lt

${ }^{\dagger}$ Univ. Lille, CNRS, UMR 8524 - Laboratoire Paul Painlevé, F-59000 Lille, France.

E-mail: charles. suquetQuniv-lille.fr 
Asymptotic properties of various extremal statistics based on the random walk $\left(S_{k}, k \geq 0\right)$ and the random field $\left(S_{k}-S_{j}, 0 \leq j<k \leq n, n \geq 1\right)$ of its increments are important from both theoretical and practical points of view and have been widely discussed in the literature. We refer to Darling, Erdös [6], Einmahl [8], Bertoin [3], Révész [21] Csörgő and Révész [5], Shao [22], Kabluchko [13], Račkauskas and Suquet[17], and the references therein for a comprehensive information on the subject.

The main object of this paper is the maximal weighted increment of the random walk $\left(S_{n}, n \geq 0\right)$ defined by

$$
T_{n}(\gamma):=\max _{0 \leq j<k \leq n} \frac{\left|S_{k}-S_{j}\right|}{(k-j)^{\gamma}}, \quad \gamma \in[0,1) .
$$

Throughout, we denote by $X$ a generic random variable which is identically distributed with each $X_{k}$ and we assume that $X$ is regularly varying with index $a>0$, denoted $X \in \mathrm{RV}_{a}$, in the sense that the tail balance condition

$$
P(X>x) \sim p x^{-a} L(x) \text { and } P(X \leq-x) \sim q x^{-a} L(x), \text { as } x \rightarrow \infty,
$$

is satisfied, where $L$ is a slowly varying function, and $p, q \in(0,1), p+q=1$. We refer to [4] for an encyclopeadic treatment of regular and slow variation. For reader's convenience, we gathered in Appendix A.4 the basics on slow variation used in this paper. In what follows, we denote

$$
X \in \mathrm{RV}_{a}(\tau) \text { if (1.1) holds with } L(x) \underset{x \rightarrow \infty}{\longrightarrow} \tau^{a}, \quad 0 \leq \tau \leq \infty .
$$

Condition (1.1) imposes a priori two requirements on the choice of $L$. First, $x^{-a} L(x)$ has to be equivalent to a nonincreasing function with limit 0 at infinity, which is automatically satisfied, see Cor.A.12. Next, $x^{-a} L(x)$ has to be equivalent to a left continuous function, which discards none slowly varying $L$ since $L$ is always equivalent to a $C^{\infty}$ function [4, Th.1.3.3]. As $L$ has not necessarily a limit at infinity, see the example built in the proof of Th.2.5, it is clear that

$$
\bigcup_{0 \leq \tau \leq \infty} \mathrm{RV}_{a}(\tau) \subsetneq \mathrm{RV}_{a}
$$

It is known that the limit distribution of $T_{n}(\gamma)$ has a threshold at $\gamma=\gamma_{a}:=1 / 2-1 / a$ for $a>2$, separating two phases named Fréchet and Brownian in the space of parameters $\{(a, \gamma) ; a>0,0<\gamma<1\}$, see Figure 1. In the Fréchet phase $\gamma \in(\max (1 / 2-1 / a, 0), 1]$ and denoting by $\underset{n \rightarrow \infty}{\stackrel{\mathcal{D}}{\longrightarrow}}$ the convergence in distribution,

$$
a_{n}^{-1} T_{n}(\gamma) \underset{n \rightarrow \infty}{\stackrel{\mathcal{D}}{\longrightarrow}} Y_{a}
$$

where $Y_{a}$ has the standard Fréchet distribution with parameter $a$, that is

$$
P\left(Y_{a} \leq x\right)=\exp \left(-x^{-a}\right), \quad x>0,
$$

and $a_{n}$ denotes the $1 / n$ quantile of $|X|$, that is

$$
a_{n}:=\inf \{x \in \mathbb{R}: P(|X| \leq x) \geq 1-1 / n\} .
$$

This result has been proved in [15]. In the Brownian phase, $0 \leq \gamma<1 / 2-1 / a$ and

$$
n^{-1 / 2+\gamma} T_{n}(\gamma) \underset{n \rightarrow \infty}{\stackrel{\mathcal{D}}{\longrightarrow}} \sigma T(\gamma):=\sigma \max _{0<|t-s| \leq 1} \frac{|W(t)-W(s)|}{|t-s|^{\gamma}},
$$

where $W=(W(t), t \in[0,1])$ is a standard Brownian motion and $\sigma^{2}=\mathrm{E} X^{2}$. This follows obviously from the Hölderian weak invariance principle proved in [16]. On the threshold $\gamma=\gamma_{a}$, the limit distribution of $T_{n}\left(\gamma_{a}\right)$ depends on the slowly varying function $L(x)$ in (1.1) and the following holds: 
Maximal weighted increment asymptotic

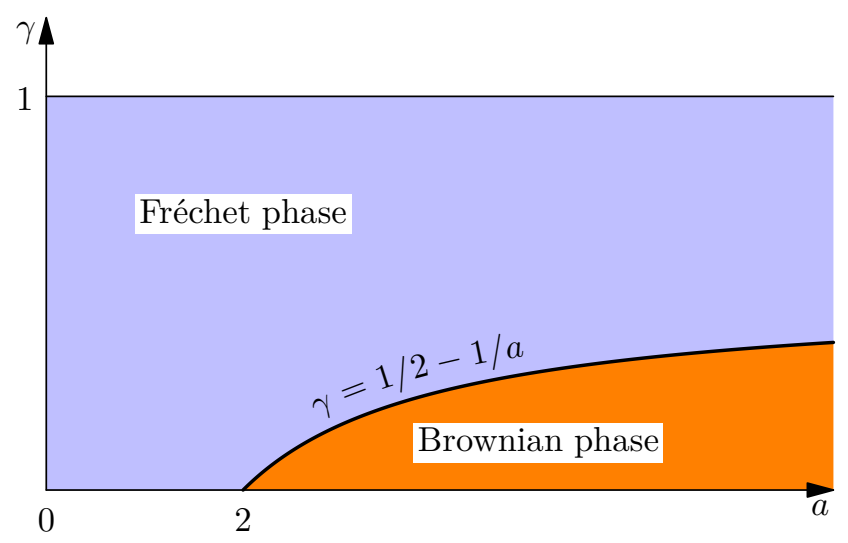

Figure 1: Fréchet and Brownian phases

- if $X \in \mathrm{RV}_{a}(\infty)$, the convergence (1.2) holds like in Fréchet phase [12, Th.5(b)];

- if $X \in \mathrm{RV}_{a}(0)$, the convergence (1.5) occurs like in Brownian phase [16].

Our main result for the case where $X \in \operatorname{RV}_{a}(\tau), a>2,0<\tau<\infty$, Theorem 2.4 below, establishes the convergence

$$
n^{-1 / a} T_{n}\left(\gamma_{a}\right) \underset{n \rightarrow \infty}{\stackrel{\mathcal{D}}{\longrightarrow}} V_{a, \sigma, \tau}:=\max \left\{\sigma T\left(\gamma_{a}\right), \tau Y_{a},\right\}
$$

where the random variables $Y_{a}$ and $T\left(\gamma_{a}\right)$ are independent. To unify the notations, we define also

$$
V_{a, \sigma, 0}:=\sigma T\left(\gamma_{a}\right), \quad V_{a, \sigma, \infty}:=Y_{a} .
$$

Up to now, no analytic formula seems to be known for the distribution of $T(\gamma)$. Fatalov [9] obtained the following exact asymptotic equivalent for the tail:

$$
P(T(\gamma)>x) \sim \frac{1}{x} \exp \left(-\frac{x^{2}}{2}\right) \frac{8 \sqrt{2}}{\sqrt{\pi}}\left(\frac{1-\gamma}{1-2 \gamma}\right)^{2}, x \rightarrow \infty, \quad 0 \leq \gamma<1 / 2 .
$$

This shows that $V_{a, \sigma, 0}$ is subgaussian (hence with light tail).

Baldi and Roynette [1, Th.4.4] have the small ball estimate

$$
\lim _{\varepsilon \rightarrow 0} \varepsilon^{2 /(1-2 \gamma)} \log P(T(\gamma) \leq \varepsilon)=-k(\gamma), \quad 0<\gamma<1 / 2,
$$

for some positive constant $k(\gamma)$.

By independence of $T\left(\gamma_{a}\right)$ and $Y_{a}$, the distribution function of $V_{a, \sigma, \tau}$ in (1.6) verifies

$$
P\left(V_{a, \sigma, \tau} \leq x\right)=P\left(\max \left\{\sigma T\left(\gamma_{a}\right), \tau Y_{a},\right\} \leq x\right)=P\left(\sigma T\left(\gamma_{a}\right) \leq x\right) \exp \left(-\tau^{a} x^{-a}\right) .
$$

In view of (1.8), this gives for $0<\tau<\infty$,

$$
\begin{aligned}
P\left(V_{a, \sigma, \tau}>x\right) & =1-\exp \left(-\tau^{a} x^{-a}\right)+P\left(\sigma T\left(\gamma_{a}\right)>x\right) \exp \left(-\tau^{a} x^{-a}\right) \\
& =\tau^{a} x^{-a}+O\left(x^{-2 a}\right), \quad x \rightarrow \infty .
\end{aligned}
$$

Hence $V_{a, \sigma, \tau}$ has a heavy tail asymptoticaly equivalent to the tail of $\tau Y_{a}$.

In the neighborhood of zero we have

$$
\lim _{\varepsilon \rightarrow 0} \varepsilon^{a} \log P\left(V_{a, \sigma, \tau} \leq \varepsilon\right)=-\sigma^{a} k\left(\gamma_{a}\right)-\tau^{a} .
$$


Roughly speaking for $0<\tau<\infty, V_{a, \sigma, \tau}$ "interpolates" (in distribution) between $V_{a, \sigma, 0}$ and $V_{a, \sigma, \infty}$. In this spirit, one may remark that

$$
\begin{aligned}
& P\left(V_{a, \sigma, \tau} \leq \sigma y\right)=P\left(T\left(\gamma_{a}\right) \leq y\right) \exp \left(\frac{-\tau^{a}}{\sigma^{a} y^{a}}\right) \\
& P\left(V_{a, \sigma, \tau} \leq \tau y\right)=P\left(T\left(\gamma_{a}\right) \leq \frac{\tau y}{\sigma}\right) \exp \left(-y^{-a}\right),
\end{aligned}
$$

whence

$$
\sigma^{-1} V_{a, \sigma, \tau} \underset{\tau \sigma^{-1} \rightarrow 0}{\stackrel{\mathcal{D}}{\longrightarrow}} T\left(\gamma_{a}\right) \text { and } \quad \tau^{-1} V_{a, \sigma, \tau} \underset{\tau \sigma^{-1} \rightarrow \infty}{\stackrel{\mathcal{D}}{\longrightarrow}} Y_{a}
$$

To sum up the case where $X \in \bigcup_{\tau \in[0, \infty]} \operatorname{RV}_{a}(\tau)$, we have the convergence

$$
b_{n}^{-1} T_{n}\left(\gamma_{a}\right) \underset{n \rightarrow \infty}{\stackrel{\mathcal{D}}{\longrightarrow}} V_{a, \sigma, \tau}, \quad \text { with } \quad b_{n}:= \begin{cases}n^{1 / a} & \text { if } \tau<\infty \\ a_{n} & \text { if } \tau=\infty\end{cases}
$$

Now it is natural to ask what happens if $X \in \mathrm{RV}_{a}$ but in none $\mathrm{RV}_{a}(\tau)$. A first answer is provided by Th.2.5 where we build an $\mathrm{RV}_{a}$ distribution for $X$ such that $\left(b_{n}^{-1} T_{n}\left(\gamma_{a}\right)\right)_{n>1}$ does not converge in distribution but has for each $\tau \in[0, \infty]$ a subsequence converging to $V_{a, \sigma, \tau}$.

Finally, we complete the picture by proving in Th.2.6 that if for some increasing positive sequence $\left(c_{n}\right)_{n \geq 1}$ and a sequence of reals $\left(\mu_{n}\right)_{n \geq 1}, c_{n}^{-1}\left(T_{n}\left(\gamma_{a}\right)-\mu_{n}\right)$ converges in distribution to some random variable $Z$ then $L$ has a limit $\tau^{a} \in[0, \infty]$ at infinity, hence $X \in \mathrm{RV}_{a}(\tau)$, and $Z=A V_{a, \sigma, \tau}+B$ in distribution for some $A>0, B \in \mathbb{R}$.

Section 2 contains the statements of our results. Theorem 2.1, Corollary 2.2 and Theorem 2.3, dealing with truncated versions of $T_{n}\left(\gamma_{a}\right)$ with respect to the length $\ell$ of increments $S_{k+\ell}-S_{k}$, are preparatory for Theorem 2.4.

The proofs are presented in Section 3. Auxiliary material and results are shifted in the Appendix section in the hope to keep a tolerable size of the proofs.

\section{Statement of results}

Throughout the paper we will need to split the range of length $\ell$ of increments $S_{k+\ell}-S_{k}$ in two or three consecutive intervals. This leads us to introduce the following generic notation for the induced blocs of weighted increments in $T_{n}(\gamma)$. We set for any real numbers $u, v$ such that $0 \leq u<v \leq n$,

$$
T_{n}^{u, v}(\gamma):=\max _{u<\ell \leq v} \max _{0 \leq k \leq n-\ell} \frac{\left|S_{k+\ell}-S_{k}\right|}{\ell^{\gamma}}
$$

As $\gamma=\gamma_{a}$ almost everywhere in the sequel, we will abbreviate $T_{n}^{u, v}\left(\gamma_{a}\right)$ as $T_{n}^{u, v}$.

From the weak invariance principle in $C[0,1]$ it follows that for any fixed $0<\theta<1$,

$$
n^{-1 / a} \sigma^{-1} T_{n}^{\theta n, n} \underset{n \rightarrow \infty}{\stackrel{\mathcal{D}}{\longrightarrow}} T^{(\theta)}(\gamma):=\max _{\theta<|t-s| \leq 1} \frac{|W(t)-W(s)|}{|t-s|^{\gamma_{a}}}
$$

see Appendix A.2 for a proof. It is proved in [15] (see Lemma 2.4 there) that for any fixed $d \geq 1$,

$$
n^{-1 / a} T_{n}^{0, d} \underset{n \rightarrow \infty}{\stackrel{\mathcal{D}}{\longrightarrow}} \tau Y_{a}
$$

Corollary 2.2 and Th. 2.3 below extend (2.2) and (2.3) to the cases where $\theta=\theta_{n} \rightarrow 0$ and $d=d_{n} \rightarrow \infty$ as $n \rightarrow \infty$ respectively.

Theorem 2.1 is used in the paper only through Corollary 2.2 but the more general formulation given here may have its own interest beyond the $\mathrm{RV}_{a}$ setting. 
Theorem 2.1. Let $X$ be a non degenerate mean zero random variable such that

$$
\mathrm{E}|X|^{b}<\infty, \quad \text { for some } b>2 .
$$

Put $\sigma^{2}:=\mathrm{E} X^{2}, \sigma>0$. Then for every $0<\gamma<1 / 2$,

$$
n^{-1 / 2+\gamma} T_{n}^{d_{n}, n} \underset{n \rightarrow \infty}{\stackrel{\mathcal{D}}{\longrightarrow}} \sigma T(\gamma)
$$

provided that with $\beta:=\min (b, 3)$,

$$
\frac{d_{n}}{n} \underset{n \rightarrow \infty}{\longrightarrow} 0 \text { and } \quad \frac{n^{1-\beta / 2} \log ^{\beta-1} n}{\left(\frac{d_{n}}{n}\right)^{\beta \gamma}} \underset{n \rightarrow \infty}{\longrightarrow} 0
$$

In particular, the convergences (2.6) are satisfied with $d_{n}=n^{\alpha} \lambda(n)$ where $\lambda$ is slowly varying and $\max \{0 ;(1-(1 / 2-\gamma) \beta) /(\beta \gamma)\}<\alpha<1$.

Corollary 2.2. Let $X$ be a non degenerate mean zero random variable whose tail function $P(|X|>x)$ is regularly varying with index $-a, a>2$. Then

$$
n^{-1 / a} T_{n}^{d_{n}, n} \underset{n \rightarrow \infty}{\stackrel{\mathcal{D}}{\longrightarrow}} \sigma T\left(\gamma_{a}\right)
$$

provided

$$
\frac{d_{n}}{n} \underset{n \rightarrow \infty}{\longrightarrow} 0 \text { and } n^{1-(2+\delta) / a} d_{n}^{(2+\delta)(1 / a-1 / 2)} \log ^{1+\delta} n \rightarrow 0 \text { as } n \rightarrow \infty
$$

with $0<\delta<a-2$, if $a \leq 3$ and $\delta=1$ if $a>3$.

If $d_{n}=c n^{\kappa}$ for $n \geq n_{0}$, then the convergence (2.7) holds if $\kappa \in\left(\frac{2(a-2-\delta)}{(2+\delta)(a-2)}, 1\right)$. The above assumption of regular variation of the tail is obviously satisfied when $X \in \mathrm{RV}_{a}$ but is more general since we do note require here the tail balance property.

Theorem 2.3. Let $a>2$ and $0<\tau<\infty$. Assume that $X \in \mathrm{RV}_{a}(\tau), \mathrm{E} X=0$ and

$$
d_{n} \rightarrow \infty, \frac{d_{n}}{n} \rightarrow 0 \text { as } n \rightarrow \infty
$$

Then

$$
n^{-1 / a} T_{n}^{0, d_{n}} \underset{n \rightarrow \infty}{\stackrel{\mathcal{D}}{\longrightarrow}} \tau Y_{a} .
$$

Theorem 2.4. Let $a>2$ and $0<\tau<\infty$. Assume that $X \in \mathrm{RV}_{a}(\tau), \mathrm{E} X=0$. Then

$$
n^{-1 / a} T_{n}\left(\gamma_{a}\right) \underset{n \rightarrow \infty}{\stackrel{\mathcal{D}}{\longrightarrow}} \max \left\{\sigma T\left(\gamma_{a}\right), \tau Y_{a},\right\}
$$

where $Y_{a}$ and $T\left(\gamma_{a}\right)$ are independent random variables, $Y_{a}$ has Fréchet distribution with parameter $a$ and $T\left(\gamma_{a}\right)$ is defined by (1.5).

Theorem 2.5. There exists a random variable $X \in \mathrm{RV}_{a} \backslash \bigcup_{\tau \in[0, \infty]} \mathrm{RV}_{a}(\tau)$ such that the following convergences hold, with limits $V_{a, \sigma, \tau}$ as in (1.6) and (1.7).

i) There is an increasing sequence of integers $\left(n_{i}\right)_{i \geq 1}$ such that

$$
n_{i}^{-1 / a} T_{n_{i}}\left(\gamma_{a}\right) \underset{i \rightarrow \infty}{\stackrel{\mathcal{D}}{\longrightarrow}} \sigma T\left(\gamma_{a}\right)=V_{a, \sigma, 0}
$$

ii) For every $\tau \in(0, \infty)$, there is an increasing sequence of integers $\left(n_{i}\right)_{i \geq 1}$ such that

$$
n_{i}^{-1 / a} T_{n_{i}}\left(\gamma_{a}\right) \underset{i \rightarrow \infty}{\stackrel{\mathcal{D}}{\longrightarrow}} \max \left\{\sigma T\left(\gamma_{a}\right), \tau Y_{a},\right\}=V_{a, \sigma, \tau}
$$


iii) There is an increasing sequence of integers $\left(n_{i}\right)_{i \geq 1}$ such that $n_{i}^{-1 / a} T_{n_{i}}\left(\gamma_{a}\right)$ is not stochastically bounded and denoting by $a_{n_{i}}$ the $1 / n_{i}$ quantile of $|X|$,

$$
a_{n_{i}}^{-1} T_{n_{i}}\left(\gamma_{a}\right) \underset{i \rightarrow \infty}{\stackrel{\mathcal{D}}{\longrightarrow}} Y_{a}=V_{a, \sigma, \infty}
$$

Theorem 2.6. Assume that $X \in \mathrm{RV}_{a}$ with $a>2$ and $\mathrm{E} X=0$ and there exists an increasing positive sequence $\left(c_{n}\right)_{n \geq 1}$ and a sequence of reals $\left(\mu_{n}\right)_{n \geq 1}$ such that

$$
c_{n}^{-1}\left(T_{n}\left(\gamma_{a}\right)-\mu_{n}\right) \underset{n \rightarrow \infty}{\stackrel{\mathcal{D}}{\longrightarrow}} Z
$$

where $Z$ is non degenerate random variable. Then the function $L$ from (1.1) has a limit $\tau^{a} \in[0, \infty]$ at infinity and there are constants $A>0, B \in \mathbb{R}$ such that

$$
Z \stackrel{d}{=} A V_{a, \sigma, \tau}+B
$$

\section{Proofs}

\subsection{Proof of Theorem 2.1}

Write

$$
T_{n}^{d_{n}, n}=\sup _{\ell, k \in I_{n}}\left|\sum_{j=1}^{n} X_{j} \delta_{j}(\ell, k)\right|,
$$

where $I_{n}=\left\{(\ell, k): d_{n} \leq \ell \leq n, 0 \leq k \leq n-\ell\right\}$, and

$$
\delta_{j}(\ell, k):= \begin{cases}\ell^{-\gamma}, & \text { if } k+1 \leq j \leq k+\ell \\ 0 & \text { otherwise. }\end{cases}
$$

In what follows, we denote by $k_{n}$ the number of elements of $I_{n}$. It is easily seen that $2 k_{n}=\left(n-\left[d_{n}\right]\right)\left(n-\left[d_{n}\right]+1\right)$, so $k_{n}<n^{2} / 2$. Let us choose and fix some enumeration of $I_{n}$. Then we introduce for $j=1, \ldots, n$, the random vector

$$
X_{n, j}=n^{-1 / 2+\gamma}\left(X_{j} \delta_{j}(\ell, k),(\ell, k) \in I_{n}\right),
$$

viewed as a random vector in $\mathbb{R}^{k_{n}}$. This leads to the representation

$$
n^{-1 / 2+\gamma} T_{n}^{d_{n}, n}=\left\|\sum_{j=1}^{n} X_{n, j}\right\|_{\infty},
$$

where $\|x\|_{\infty}=\max _{1 \leq i \leq k_{n}}\left|x_{k}\right|, x=\left(x_{k}\right) \in \mathbb{R}^{k_{n}}$ and dimension $k_{n} \leq n^{2}$.

Let $Y, Y_{k}, k \geq 1$, be i.i.d. Gaussian random variables, such that $\mathrm{E} Y=0, \mathrm{E} Y^{2}=\mathrm{E} X^{2}$. Following Lindeberg method, we substitute step by step each $X_{n, j}$ by $Y_{n, j}$ where $Y_{n, j}=$ $n^{-1 / 2+\gamma}\left(Y_{j} \delta_{j}(\ell, k),(\ell, k) \in I_{n}\right) \in \mathbb{R}^{k_{n}}$, in order to compare the distribution of $T_{n}^{d_{n}, n}$ with that of

$$
\widehat{T}_{n}^{d_{n}, n}:=\max _{d_{n}<\ell \leq n} \ell^{-\gamma} \max _{0 \leq k \leq n-\ell}\left|Y_{k+1}+\cdots+Y_{k+\ell}\right|=\left\|\sum_{j=1}^{n} Y_{n, j}\right\|_{\infty} .
$$

To this aim we consider for each $r>0$ and $\varepsilon>0$ the function $f_{r, \varepsilon}: \mathbb{R}^{k_{n}} \rightarrow \mathbb{R}$ which enjoys the following two properties:

(i) for each $x \in \mathbb{R}^{k_{n}}$,

$$
\mathbb{1}\left\{\|x\|_{\infty} \leq r\right\} \leq f_{r, \varepsilon}(x) \leq \mathbb{1}\left\{\|x\|_{\infty} \leq r+\varepsilon\right\}
$$


(ii) the function $f_{r, \varepsilon}$ is three times differentiable and for each $m=1,2,3$, there exists an absolute constant $c_{m}>0$ such that, recalling that $k_{n}+1<n^{2}$,

$$
\left\|f_{r, \varepsilon}^{(m)}\right\|:=\sup \left\{\left|f_{r, \varepsilon}^{(m)}(x)(h)^{m}\right|: x, h \in \mathbb{R}^{k_{n}},\|h\|_{\infty} \leq 1\right\} \leq c_{m} \varepsilon^{-m} \log ^{m-1} n .
$$

Here $f_{r, \varepsilon}^{(m)}$ denotes the $m^{\text {th }}$ derivative of the function $f_{r, \varepsilon}$ and $f_{r, \varepsilon}^{(m)}(x)(h)^{m}$ the corresponding differential. Such functions are constructed in Bentkus [2].

By using (i), we have

$$
\begin{aligned}
P\left(n^{-1 / 2+\gamma} T_{n}^{d_{n}, n} \leq r\right)=P\left(\left\|\sum_{j=1}^{n} X_{n, j}\right\|_{\infty} \leq r\right) & \leq \mathrm{E} f_{r, \varepsilon}\left(\sum_{i=1}^{n} X_{n, i}\right) \\
& =\Delta(n, \varepsilon)+\mathrm{E} f_{r, \varepsilon}\left(\sum_{i=1}^{n} Y_{n, i}\right) \\
& \leq \Delta(n, \varepsilon)+P\left(n^{-1 / 2+\gamma} \widehat{T}_{n}^{\left(d_{n}\right)} \leq r+\varepsilon\right),
\end{aligned}
$$

where

$$
\Delta(n, \varepsilon):=\mathrm{E} f_{r, \varepsilon}\left(\sum_{i=1}^{n} X_{n, i}\right)-\mathrm{E} f_{r, \varepsilon}\left(\sum_{i=1}^{n} Y_{n, i}\right) .
$$

Now, we introduce the hybrid sums $Z_{n, j}$ with hole at index $j$,

$$
Z_{n, j}:=\sum_{1 \leq i<j} X_{n, i}+\sum_{j<i \leq n} Y_{n, i}, \quad j=0, \ldots, n, n+1
$$

with the convention $\sum_{i \in \emptyset}:=0$. In particular $\sum_{i=1}^{n} X_{n, i}=Z_{n, n+1}$ and $\sum_{i=1}^{n} Y_{n, i}=Z_{n 0}$. Defining $X_{n 0}:=0$ and $Y_{n, n+1}:=0$, we notice also that

$$
Z_{n, j}+Y_{n, j}=Z_{n, j-1}+X_{n, j-1}, \quad j=1, \ldots, n+1 .
$$

With these notations, we can describe the progressive substitution of the $X_{n, j}$ 's by the $Y_{n, j}$ 's as

$$
\begin{aligned}
f_{r, \varepsilon}\left(\sum_{i=1}^{n} X_{n, i}\right)-f_{r, \varepsilon}\left(\sum_{i=1}^{n} Y_{n, i}\right) & =f_{r, \varepsilon}\left(Z_{n, n+1}\right)-f_{r, \varepsilon}\left(Z_{n, 0}\right) \\
& =\sum_{j=1}^{n}\left(f_{r, \varepsilon}\left(Z_{n, j}+X_{n, j}\right)-f_{r, \varepsilon}\left(Z_{n, j-1}+X_{n, j-1}\right)\right) \\
& =\sum_{j=1}^{n}\left(f_{r, \varepsilon}\left(Z_{n, j}+X_{n, j}\right)-f_{r, \varepsilon}\left(Z_{n, j}+Y_{n, j}\right)\right) .
\end{aligned}
$$

whence

$$
\Delta(n, \varepsilon)=\sum_{j=1}^{n}\left(\mathrm{E} f_{r, \varepsilon}\left(Z_{n, j}+X_{n, j}\right)-\mathrm{E} f_{r, \varepsilon}\left(Z_{n, j}+Y_{n, j}\right)\right) .
$$

Next we shall use Taylor's expansion

$$
f_{r, \varepsilon}(x+h)=f_{r, \varepsilon}(x)+f_{r, \varepsilon}^{\prime}(x)(h)+2^{-1} f_{r, \varepsilon}^{\prime \prime}(x)(h, h)+R
$$

with interpolated remainder, see Lemma A.1 in Appendix A.1,

$$
|R| \leq 6^{2-\beta}\left\|f_{r, \varepsilon}^{\prime \prime}\right\|^{3-\beta}\left\|f_{r, \varepsilon}^{\prime \prime \prime}\right\|^{\beta-2}\|h\|_{\infty}^{\beta} \leq 6^{2-\beta} c_{2}^{3-\beta} c_{3}^{\beta-2} \varepsilon^{-\beta}\|h\|_{\infty}^{\beta} \log ^{\beta-1} n
$$


valid for any $2<\beta \leq 3$. Recalling that $\mathrm{E}\left|X_{j}\right|^{b}<\infty$ for some $b>2$, we choose from now on $\beta=\min (b, 3)$. For each $j=1, \ldots, n$, the random vectors $Z_{n, j}$ and $X_{n, j}$ of $\mathbb{R}^{k_{n}}$ are independent and the same holds for $Z_{n, j}$ and $Y_{n, j}$. By Lemma A.2 in Appendix A.1, this implies $\mathrm{E} f_{r, \varepsilon}^{\prime}\left(Z_{n, j}\right)\left(X_{n, j}\right)=\left(\mathrm{E}\left(f_{r, \varepsilon}^{\prime}\left(Z_{n, j}\right)\right)\left(\mathrm{E} X_{n, j}\right)\right.$ and similarly with $Y_{n, j}$ instead of $X_{n, j}$. As $X_{n, j}$ and $Y_{n, j}$ have null expectation, this gives

$$
\mathrm{E} f_{r, \varepsilon}^{\prime}\left(Z_{n, j}\right)\left(X_{n, j}\right)=\mathrm{E} f_{r, \varepsilon}^{\prime}\left(Z_{n, j}\right)\left(Y_{n, j}\right)=0 .
$$

As moreover $X_{n, j}$ and $Y_{n, j}$ have the same covariance matrix, Lemma A.3 gives also

$$
\mathrm{E} f_{r, \varepsilon}^{\prime \prime}\left(Z_{n, j}\right)\left(X_{n, j}, X_{n, j}\right)=\mathrm{E} f_{r, \varepsilon}^{\prime \prime}\left(Z_{n, j}\right)\left(Y_{n, j}, Y_{n, j}\right) .
$$

Now applying Taylor's formula (3.4) to each term in (3.3) and accounting (3.5) gives

$$
|\Delta(n, \varepsilon)| \leq\left(6^{2-\beta} c_{2}^{3-\beta} c_{3}^{\beta-2}\right) \varepsilon^{-\beta}\left(\log ^{\beta-1} n\right) \sum_{j=1}^{n}\left[\mathrm{E}\left\|X_{n, j}\right\|_{\infty}^{\beta}+\mathrm{E}\left\|Y_{n, j}\right\|_{\infty}^{\beta}\right] .
$$

Noticing that $X_{n, j}$ is the product of the (scalar) real random variable $X_{j}$ by the deterministic vector $\left(n^{-1 / 2+\gamma} \delta_{j}(\ell, k),(\ell, k) \in I_{n}\right)$ of $\mathbb{R}^{k_{n}}$, it is clear that

$$
\left\|X_{n, j}\right\|_{\infty}=n^{-1 / 2+\gamma} \max _{(\ell, k) \in I_{n}} \delta_{j}(\ell, k)\left|X_{j}\right|=n^{-1 / 2+\gamma} d_{n}^{-\gamma}\left|X_{j}\right|,
$$

whence,

$$
\mathrm{E}\left\|X_{n, j}\right\|_{\infty}^{\beta}=n^{(-1 / 2+\gamma) \beta} d_{n}^{-\beta \gamma} \mathrm{E}\left|X_{j}\right|^{\beta}, \quad \mathrm{E}\left\|Y_{n, j}\right\|_{\infty}^{\beta}=n^{(-1 / 2+\gamma) \beta} d_{n}^{-\beta \gamma} \mathrm{E}\left|Y_{j}\right|^{\beta} .
$$

Therefore, puting $C_{\beta}:=6^{2-\beta} c_{2}^{3-\beta} c_{3}^{\beta-2}\left(\mathrm{E}|X|^{\beta}+\mathrm{E}|Y|^{\beta}\right)$,

$$
|\Delta(n, \varepsilon)| \leq C_{\beta} \varepsilon^{-\beta} n^{1+(-1 / 2+\gamma) \beta} d_{n}^{-\beta \gamma} \log ^{\beta-1} n=C_{\beta} \varepsilon^{-\beta} \frac{n^{1-\beta / 2} \log ^{\beta-1} n}{\left(\frac{d_{n}}{n}\right)^{\beta \gamma}} .
$$

By condition (2.6), $\Delta(n, \varepsilon) \rightarrow 0$ as $n \rightarrow \infty$ and we find from (3.1), for each $\varepsilon>0$,

$$
\limsup _{n \rightarrow \infty} P\left(n^{-1 / 2+\gamma} T_{n}^{d_{n}, n} \leq r\right) \leq \limsup _{n \rightarrow \infty} P\left(n^{-1 / 2+\gamma} \widehat{T}_{n}^{d_{n}, n} \leq r+\varepsilon\right) .
$$

From weak invariance principle in Hölder spaces, see [16] and Appendix A.2 below,

$$
n^{-1 / 2+\gamma} \widehat{T}_{n}^{d_{n}, n}(\gamma) \underset{n \rightarrow \infty}{\stackrel{\mathcal{D}}{\longrightarrow}} T(\gamma),
$$

for any sequence $d_{n}$ such that $d_{n} / n \rightarrow 0$ as $n \rightarrow \infty$. Since the distribution function of $T(\gamma)$ is continuous, the limsup in the right-hand side of (3.9) is a true limit equal to $P(T(\gamma) \leq r+\varepsilon)$ and we obtain

$$
\limsup _{n \rightarrow \infty} P\left(n^{-1 / 2+\gamma} T_{n}^{d_{n}, n} \leq r\right) \leq P(T(\gamma) \leq r+\varepsilon) .
$$

To find a lower bound for $\liminf _{n \rightarrow \infty} P\left(n^{-1 / 2+\gamma} T_{n}^{d_{n}, n} \leq r\right)$, we consider $\varepsilon>0$ such that $0<\varepsilon<r$ and the function $f_{r-\varepsilon, \varepsilon}$, which gives

$$
\begin{aligned}
P\left(n^{-1 / 2+\gamma} T_{n}^{d_{n}, n} \leq r\right) & =P\left(\left\|\sum_{j=1}^{n} X_{n, j}\right\|_{\infty} \leq r\right) \geq \mathrm{E} f_{r-\varepsilon, \varepsilon}\left(\sum_{i=1}^{n} X_{n, i}\right) \\
& =\Delta^{\prime}(n, \varepsilon)+\mathrm{E} f_{r-\varepsilon, \varepsilon}\left(\sum_{i=1}^{n} Y_{n, i}\right) \\
& \geq-\left|\Delta^{\prime}(n, \varepsilon)\right|+P\left(n^{-1 / 2+\gamma} \widehat{T}_{n}^{d_{n}, n} \leq r+\varepsilon\right),
\end{aligned}
$$


where

$$
\Delta^{\prime}(n, \varepsilon):=\mathrm{E} f_{r-\varepsilon, \varepsilon}\left(\sum_{i=1}^{n} X_{n, i}\right)-\mathrm{E} f_{r-\varepsilon, \varepsilon}\left(\sum_{i=1}^{n} Y_{n, i}\right) .
$$

Acting as above we estimate

$$
\left|\Delta^{\prime}(n, \varepsilon)\right| \leq C_{\beta} \varepsilon^{-\beta} n^{1+(-1 / 2+\gamma) \beta} d_{n}^{-\beta \gamma} \log ^{\beta-1} n,
$$

which gives

$$
\liminf _{n \rightarrow \infty} P\left(n^{-1 / 2+\gamma} T_{n}^{d_{n}, n} \leq r\right) \geq \liminf _{n \rightarrow \infty} P\left(n^{-1 / 2+\gamma} \widehat{T}_{n}^{d_{n}, n} \leq r-\varepsilon\right)=P(T(\gamma) \leq r-\varepsilon) .
$$

Combining with (3.11) we have

$$
\begin{aligned}
P(T(\gamma) \leq r-\varepsilon) & \leq \liminf _{n \rightarrow \infty} P\left(n^{-1 / 2+\gamma} T_{n}^{d_{n}, n} \leq r\right) \\
& \leq \limsup _{n \rightarrow \infty} P\left(n^{-1 / 2+\gamma} T_{n}^{d_{n}, n} \leq r\right) \leq P(T(\gamma) \leq r+\varepsilon) .
\end{aligned}
$$

Since the distribution function of $T(\gamma)$ is continuous, see the Appendix A.3, the proof is completed by letting $\varepsilon \rightarrow 0$ in (3.12).

Proof of Corollary 2.2. If the tail function of $|X|$ is regularly varying with index $-a, a>2$, then $\mathrm{E}|X|^{b}<\infty$ for any $0 \leq b<a$. As $a>2$, we can choose $2<b<a$ and apply Th.2.2 with $\beta=\min (3, b)=2+\delta$ which gives the result.

\subsection{On $L$-subsequences}

Before presenting the proof of theorems 2.3 and 2.4, it seems convenient to make some remarks on the use of subsequences in this paper. In the first part of our contribution, i.e. until Th. 2.4, we obtain the limiting behavior of $T_{n}\left(\gamma_{a}\right)$ for $X \in \bigcup_{0 \leq \tau \leq \infty} \operatorname{RV}_{a}(\tau)$. Next we investigate the complementary case where $L$ has no limit at all at infinity. Our main tool is then the exploitation of some $L$-subsequences versions of all the convergence theorems leading to Th.4, see for instance the subsequence version of Hölderian invariance principle, A.2-Th.A.4. By $L$-subsequence we mean a subsequence indexed by an infinite subset $I$ of $\mathbb{N}$, whose construction depends on the asymptotic behavior of $L$. Our main example is $I$ such that

$$
L\left(n^{1 / a}\right) \underset{n \rightarrow \infty, n \in I}{\longrightarrow} \tau^{a}, \quad \text { for some } \tau \in[0, \infty] .
$$

The convergence of $\left(n^{-1 / a} T_{n}\left(\gamma_{a}\right)\right)_{n \in I}$ cannot be inherited from the whole sequence since (3.13) is weaker in general than $X \in \mathrm{RV}_{a}(\tau)$. This announces the tedious task of a careful rereading of the proofs of Th.2.1 to Th.2.4 and also of the convergences results for the special cases $\tau=0, \tau=\infty$ to check if an adaptation to some $L$-subsequence is possible. In order to minimize such a burden, we will write the forthcoming proofs of the Theorems 2.3 and 2.4 for a generic subsequence indexed by an infinite subset $I$ of $\mathbb{N}$, replacing the hypothesis $L(x) \rightarrow \tau^{a}$ by (3.13). Then the proof of the theorem will just be the special case where $I=\mathbb{N}$, where (3.13) is automaticaly satisfied when $X \in \mathrm{RV}_{a}(\tau)$. The reader interested by the proof of Th. 2.4 only, can ignore the mention $n \in I$ everywhere in the proof. To have minor modifications in the typesetting we adopt the notation $\underset{n \rightarrow \infty, n \in I}{\longrightarrow}$ which avoids double indexing by $n_{i}$.

Before proceeding, let us remark that Th.2.1 and Cor.2.2 remain valid for any $L$ subsequence because they are established under the hypothesis $\mathrm{E}|X|^{b}<\infty$ for some $2<b<a$ which does not involve $L$ at all. 


\subsection{Proof of Theorem 2.3}

The following auxiliary results are used in the proof.

Lemma 3.1. Let $\left(Y_{i}\right)$ be a sequence of i.i.d. random variables. Then, for any $b>0, \gamma \geq 0$, $h \geq 1$ and $H \leq n$,

$$
\begin{aligned}
& P\left(\max _{h \leq \ell \leq H} \ell^{-\gamma} \max _{0 \leq k \leq n-\ell}\left|\sum_{j=k+1}^{k+\ell} Y_{j}\right|>b\right) \leq 2 \sum_{n / H \leq 2^{j} \leq n / h} Q_{j}, \\
& Q_{j}:=2^{j} P\left(\max _{1 \leq k \leq 2 n 2^{-j}}\left|\sum_{j=1}^{k} Y_{j}\right|>b\left(n 2^{-j}\right)^{\gamma}\right) .
\end{aligned}
$$

This lemma is proved in [15], see Lemma 3.3 therein. The next one extends and completes Lemma 2.4 in [19]. In view of its role in this current work, we provide a detailed proof below.

Lemma 3.2. Assume that $X$ satisfies for some $a>1$ and some slowly varying $L$,

$$
G(x):=P(|X|>x) \sim x^{-a} L(x), \quad x \rightarrow \infty .
$$

Denote by $a_{n}$ the $1-1 / n$ quantile defined by (1.4) and by $\left(c_{n}\right)_{n \geq 1}$ a nondecreasing sequence of positive reals such that

$$
P\left(|X|>c_{n}\right) \sim n^{-1}, \quad n \rightarrow \infty .
$$

i) For any $0<s<a$,

$$
\mathrm{E}|X|^{s} \mathbb{1}_{\left\{|X|>y c_{n}\right\}} \leq \frac{2 a}{a-s} c_{n}^{s} n^{-1} y^{s-a},
$$

for $n$ large enough, uniformly in $y \in[1, \infty)$.

ii) For any $s>a$,

$$
\mathrm{E}|X|^{s} \mathbb{1}_{\left\{|X| \leq y c_{n}\right\}} \leq \frac{2 a}{s-a} c_{n}^{s} n^{-1} y^{s-a},
$$

for $n$ large enough, uniformly in $y \in[1, \infty)$.

iii) The inequalities (3.16) and (3.17) remain valid if we replace $c_{n}$ by $b_{n} \sim a_{n}$. Moreover if $\left(c_{n}\right)_{n \geq 1}$ satisfies (3.15), then $c_{n} \sim a_{n}$ as $n \rightarrow \infty$.

iv) $a_{n}=n^{1 / a} l(n)$ where $l$ is a slowly varying function.

Proof. By (3.14), $\widetilde{L}(x):=x^{a} G(x) \sim L(x)$ hence $\widetilde{L}$ is slowly varying and $G(x)=x^{-a} \widetilde{L}(x)$ is regularly varying with exponent $-a$.

To prove i), a Fubini argument gives with the notations $G_{s}$ and $G_{s}^{*}$ defined by (A.19),

$$
\begin{aligned}
\mathrm{E}|X|{ }^{s} \mathbb{1}_{\left\{|X|>y c_{n}\right\}} & =\left(y c_{n}\right)^{s} P\left(|X|>y c_{n}\right)+s \int_{y c_{n}}^{\infty} t^{s-1} P(|X|>t) \mathrm{d} t \\
& =\left(y c_{n}\right)^{s} G\left(y c_{n}\right)+s G_{s-1}^{*}\left(y c_{n}\right) .
\end{aligned}
$$

By Karamata theorem, see (A.20) in A.4-Th.A.13 with $\lambda=-(s-1-a+1)=a-s>0$,

$$
\frac{G_{s-1}^{*}\left(y c_{n}\right)}{\left(y c_{n}\right)^{s} G\left(y c_{n}\right)} \underset{n \rightarrow \infty}{\longrightarrow} \frac{1}{\lambda}=\frac{1}{a-s} .
$$

For $y \geq 1, c_{n} y \geq c_{n} \rightarrow \infty$, so the above convergence is obviously uniform in $y \in[1, \infty)$. Hence when $n$ tends to infinity,

$$
\mathrm{E}|X|{ }^{s} \mathbb{1}_{\left\{|X|>y c_{n}\right\}} \sim \frac{a}{a-s}\left(y c_{n}\right)^{s} G\left(y c_{n}\right),
$$


uniformly in $y \in[1, \infty)$. Now since $G$ has regular variation with exponent $-a<0$, $G\left(y c_{n}\right) \sim y^{-a} G\left(c_{n}\right)$, uniformly in $y \in[1, \infty)$, see e.g. Th.1.5.2 in [4]. Accounting (3.15),

$$
\mathrm{E}|X|^{s} \mathbb{1}_{\left\{|X|>y c_{n}\right\}} \sim \frac{a}{a-s} c_{n}^{s} G\left(c_{n}\right) y^{s-a} \sim \frac{a}{a-s} c_{n}^{s} n^{-1} y^{s-a},
$$

uniformly in $y \in[1, \infty)$. Introducing the extra factor 2 to replace this equivalence by an inequality gives (3.16) valid for $n$ large enough uniformly in $y \in[1, \infty)$.

The proof of ii) is completely similar and will be omitted.

To prove iii), we note first that an obvious choice is $c_{n}=a_{n}$. If we replace $c_{n}$ by $b_{n}$ in the proof of i), everything works identically until the first equivalence in (3.19), noting that the equivalence $a_{n} \sim b_{n}$ implies that $b_{n}$ tends to infinity. To obtain the second equivalence in (3.19) leading to (3.16), we note that $L\left(a_{n}\right) \sim L\left(b_{n}\right)$ by A.4-Cor.A.10 ii), so $G\left(b_{n}\right) \sim b_{n}^{-a} L\left(b_{n}\right) \sim a_{n}^{-a} L\left(a_{n}\right) \sim G\left(a_{n}\right)=n^{-1}$.

To check the second assertion in iii), we use the fact that (3.15) and (3.16) imply

$$
c_{n}^{-1} \max _{1 \leq k \leq n}\left|X_{k}\right| \underset{n \rightarrow \infty}{\stackrel{\mathcal{D}}{\longrightarrow}} Y_{a} .
$$

Indeed if $F$ and $F_{n}$ are the distribution functions of $|X|$ and $\max _{1 \leq k \leq n}\left|X_{k}\right|, F_{n}=F^{n}$ and with some function $u(x) \rightarrow 1$ at infinity and for any fixed $x>0$,

$$
F_{n}\left(c_{n} x\right)=\left(1-u\left(c_{n} x\right)\left(c_{n} x\right)^{-a} L\left(c_{n} x\right)\right)^{n}=\exp \left(n \log \left(1-u\left(c_{n} x\right) \frac{L\left(c_{n} x\right)}{c_{n}^{a} x^{a}}\right)\right) .
$$

As $n \rightarrow \infty, u\left(c_{n} x\right) c_{n}^{-a} x^{-a} L\left(c_{n} x\right) \sim c_{n}^{-a} x^{-a} L\left(c_{n}\right) \sim x^{-a} P\left(|X|>c_{n}\right) \sim x^{-a} n^{-1}$, whence $\lim _{n \rightarrow \infty} F_{n}\left(c_{n} x\right)=\exp \left(-x^{-a}\right)=P\left(Y_{a} \leq x\right)$. Now the weak convergence (3.20) works with the two sequences of normalizing constants $\left(c_{n}\right)_{n \geq 1}$ and $\left(a_{n}\right)_{n \geq 1}$, so by the convergence of types, see [11, Th.1, 2, p.40-42] or [20, Prop.0.2], there is a constant $A>0$ such that $c_{n} / a_{n}$ converges to 1 and $P\left(Y_{a} \leq x\right)=P\left(Y_{a} \leq A x\right)$, so $A=1$, that is $c_{n} \sim a_{n}$.

To prove iv), we first recall that the left continuous inverse of a non decreasing function $H(x)$ is $H^{\leftarrow}(y):=\inf \{x: H(x) \geq y\}$. In particular it is easily seen that $a_{n}=$ $(1 / G)^{\leftarrow}(n)$, noticing that the slowly varying $L$ in (3.14) is positive on some neighborhood of infinity which forbid $G$ to vanish at some real $x$. Now $1 / G(x)=x^{a} / \widetilde{L}(x)$, so $1 / G$ varies regularly with exponent $a$. By [20, Prop.0.8(v) p.23], this entails that $(1 / G)^{\leftarrow}$ varies regularly with exponent $1 / a$.

Proof of Th. 2.3. Let $X$ be in $\mathrm{RV}_{a}$ and $I$ be an infinite subset of $\mathbb{N}$ such that

$$
L\left(n^{1 / a}\right) \underset{n \rightarrow \infty, n \in I}{\longrightarrow} \tau^{a} .
$$

We prove the weak convergence of $\left(n^{-1 / a} T_{n}^{0, d_{n}}\right)_{n \in I}$ to $\tau Y_{a}$ under (2.9) restricted to $I$ and (3.21). Th. 2.3 follows when $X \in \mathrm{RV}_{a}(\tau)$, since this membership allows the choice $I=\mathbb{N}$ in (3.21).

By [15, Lem.2.4] for fixed $d \geq 1,\left(a_{n}^{-1} T_{n}^{0, d}\right)_{n \in \mathbb{N}}$ converges to $Y_{a}$ in distribution. Using (3.21), one sees that $P\left(|X|>\tau n^{1 / a}\right) \sim n^{-1}$, as $n \rightarrow \infty, n \in I$. Hence by Lemma 3.2, $a_{n} \sim \tau n^{1 / a}$ as $n \rightarrow \infty, n \in I$, whence $\left(n^{-1 / a} T_{n}^{0, d}\right)_{n \in I}$ converges to $\tau Y_{a}$. So by Lemma 3.1, we only need to prove that for each $\varepsilon>0$,

$$
\lim _{h \rightarrow \infty} \limsup _{n \rightarrow \infty, n \in I} Q_{n}(h, \varepsilon)=0
$$

where

$$
Q_{n}(h, \varepsilon):=P\left(\max _{h \leq \ell \leq d_{n}} \ell^{-\gamma_{a}} \max _{0 \leq k \leq n-\ell}\left|S_{k+\ell}-S_{k}\right|>\varepsilon n^{1 / a}\right) .
$$


Until the end of the proof, $n$ belongs to $I$. Consider the truncated random variables

$$
X_{i}^{\prime}=X_{i} \mathbb{1}\left\{\left|X_{i}\right| \leq \tau h^{\gamma_{a}} n^{1 / a}\right\}, \quad \widetilde{X}_{i}=X_{i}^{\prime}-\mathrm{E}\left(X_{i}^{\prime}\right), i=1, \ldots, n, n \in I
$$

and the corresponding partial sums

$$
S_{k}^{\prime}=\sum_{i=1}^{k} X_{i}^{\prime} \text { and } \widetilde{S}_{k}=\sum_{i=1}^{k} \widetilde{X}_{i}, \quad k \geq 1
$$

with $S_{0}^{\prime}=\widetilde{S}_{0}=0$. From Lemma 3.1, we conclude

$$
\begin{aligned}
Q_{n}(h, \varepsilon) & \leq P\left(\max _{1 \leq k \leq n}\left|X_{k}\right| \geq \tau h^{\gamma_{a}} n^{1 / a}\right)+P\left(\max _{h \leq \ell \leq d_{n}} \ell^{-\gamma_{a}} \max _{0 \leq k \leq n-\ell}\left|S_{k+\ell}^{\prime}-S_{k}^{\prime}\right|>\varepsilon n^{1 / a}\right) \\
& \leq P\left(\max _{1 \leq k \leq n}\left|X_{k}\right| \geq \tau h^{\gamma_{a}} n^{1 / a}\right)+2 \sum_{n / d_{n} \leq 2^{j} \leq n / h} 2^{j} Q_{n, j}(h, \varepsilon),
\end{aligned}
$$

where

$$
Q_{n, j}(h, \varepsilon):=P\left(\max _{1 \leq k \leq 2 n 2^{-j}}\left|S_{k}^{\prime}\right|>\varepsilon\left(n 2^{-j}\right)^{\gamma_{a}} n^{1 / a}\right)
$$

Since

$$
\lim _{n \rightarrow \infty, n \in I} P\left(\max _{1 \leq k \leq n}\left|X_{k}\right|>\tau h^{\gamma_{a}} n^{1 / a}\right)=1-\exp \left(-h^{-\gamma_{a} a}\right) \underset{h \rightarrow \infty}{\longrightarrow} 0,
$$

the proof of (3.22) reduces to

$$
\lim _{h \rightarrow \infty} \limsup _{n \rightarrow \infty, n \in I} \sum_{n / d_{n} \leq 2^{j} \leq n / h} 2^{j} Q_{n, j}(h, \varepsilon)=0 .
$$

By Lemma 3.2 (i) applied with $c_{n}=\tau n^{1 / a}, n \in I$ and noticing that because $\mathrm{E} X=0$,

$$
\mathrm{E} X^{\prime}=\mathrm{E}\left(X-X \mathbb{1}\left\{|X|>\tau h^{\gamma_{a}} n^{1 / a}\right\}\right)=-\mathrm{E}\left(X \mathbb{1}\left\{|X|>\tau h^{\gamma_{a}} n^{1 / a}\right\}\right)
$$

we have

$$
\left|\mathrm{E}\left(S_{k}^{\prime}\right)\right| \leq k\left|\mathrm{E}\left(X^{\prime}\right)\right| \leq k \mathrm{E}\left(|X| \mathbb{1}\left\{|X|>\tau h^{\gamma_{a}} n^{1 / a}\right\}\right) \leq k \frac{2 a \tau}{a-1} n^{-1+1 / a} h^{\gamma_{a}(1-a)} .
$$

This yields

$$
\max _{1 \leq k \leq 2 n 2^{-j}}\left|S_{k}^{\prime}\right| \leq \max _{1 \leq k \leq 2 n 2^{-j}}\left|\widetilde{S}_{k}\right|+2^{-j} \frac{4 a \tau}{a-1} n^{1 / a} h^{\gamma_{a}(1-a)} .
$$

Moreover as $j \leq \log _{2}(n / h)$, we have $2^{-j} \leq 1 \leq\left(n 2^{-j}\right)^{\gamma_{a}}$ since $\gamma_{a} \geq 0$. Hence for $h$ large enough and uniformly in $j \leq \log _{2}(n / h)$,

$$
\max _{1 \leq k \leq 2 n 2^{-j}}\left|S_{k}^{\prime}\right| \leq \max _{1 \leq k \leq 2 n 2^{-j}}\left|\widetilde{S}_{k}\right|+\frac{\varepsilon}{2}\left(n 2^{-j}\right)^{\gamma_{a}} n^{1 / a}
$$

Hence, for $h$ large enough and uniformly in $j$ such that $2^{j} \leq n / h$,

$$
Q_{n, j}(h, \varepsilon) \leq \widetilde{Q}_{n, j}(h, \varepsilon),
$$

where

$$
\widetilde{Q}_{n, j}(h, \varepsilon):=P\left(\max _{1 \leq k \leq 2 n 2^{-j}}\left|\widetilde{S}_{k}\right|>(\varepsilon / 2)\left(n 2^{-j}\right)^{\gamma_{a}} n^{1 / a}\right) .
$$

Fix $p>a$. Since $\left(\left|\widetilde{S}_{k}\right|, k \geq 1\right)$ is a submartingale, by Doob and Markov inequalities we obtain

$$
\widetilde{Q}_{n, j}(h, \varepsilon) \leq\left(\frac{p}{p-1}\right)^{p}\left(\frac{2}{\varepsilon}\right)^{p}\left(\left(n 2^{-j}\right)^{\gamma_{a}} n^{1 / a}\right)^{-p} \mathrm{E}\left|\widetilde{S}_{2 n 2^{-j}}\right|^{p} .
$$


By Rosenthal inequality,

$\mathrm{E}\left|\widetilde{S}_{n 2^{-j}}\right|^{p} \leq c_{p}\left[\left(n 2^{-j}\right)^{p / 2}\left(\mathrm{E}\left(|X|^{2} \mathbb{1}\left\{|X| \leq \tau n^{1 / a} h^{\gamma_{a}}\right\}\right)^{p / 2}+n 2^{-j} \mathrm{E}|X|^{p} \mathbb{1}\left\{|X| \leq \tau n^{1 / a} h^{\gamma_{a}}\right\}\right]\right.$, where the constant $c_{p}>0$ depends on $p$ only. Applying Lemma 3.2, we obtain

$$
\mathrm{E}\left|\widetilde{S}_{n 2^{-j}}\right|^{p} \leq c_{p}\left[\left(n 2^{-j}\right)^{p / 2}\left(\mathrm{E} X^{2}\right)^{p / 2}+n 2^{-j} \frac{2 a \tau^{p}}{p-a} n^{p / a} n^{-1} h^{\gamma_{a}(p-a)}\right] .
$$

This leads to

$$
\begin{aligned}
\widetilde{Q}_{n j}(h, \varepsilon) & \leq C_{p}\left(\frac{2}{\varepsilon}\right)^{p}\left[\left(n 2^{-j}\right)^{\gamma_{a}} n^{1 / a}\right]^{-p}\left[\left(n 2^{-j}\right)^{p / 2} \sigma^{p}+n 2^{-j} \frac{2 a \tau^{p}}{p-a} n^{p / a} n^{-1} h^{\gamma_{a}(p-a)}\right] \\
& =C_{p}\left(\frac{2}{\varepsilon}\right)^{p}\left[\sigma^{p} n^{-p / a}\left(n 2^{-j}\right)^{-\gamma_{a} p+p / 2}+\frac{2 a \tau^{p}}{p-a} n^{-1} h^{\gamma_{a}(p-a)}\left(n 2^{-j}\right)^{-\gamma_{a} p+1}\right],
\end{aligned}
$$

where $C_{p}:=(1-1 / p)^{-p} c_{p}$ and $\sigma^{2}=\mathrm{E} X^{2}$. Hence, recalling that $\gamma_{a}=1 / 2-1 / a$ and $p>a$, we obtain

$$
\begin{aligned}
& \sum_{n / d_{n} \leq 2^{j} \leq n / h} 2^{j} Q_{n j}(h, \varepsilon) \leq \sum_{n / d_{n} \leq 2^{j} \leq n / h} 2^{j} \widetilde{Q}_{n j}(h, \varepsilon) \\
\leq & C_{p}\left(\frac{2}{\varepsilon}\right)^{p}\left[\sigma^{p} n^{p / a} n^{-p / a} \sum_{n / d_{n} \leq 2^{j} \leq n / h} 2^{-(p / a-1) j}+\frac{2 a \tau^{p}}{p-a} n^{-\gamma_{a} p} h^{\gamma_{a}(p-a)} \sum_{n / d_{n} \leq 2^{j} \leq n / h} 2^{\gamma_{a} p j}\right] \\
\leq & C_{p}\left(\frac{2}{\varepsilon}\right)^{p}\left[\frac{2^{p / a}}{2^{p / a}-2} \sigma^{p} K_{n}+\frac{2^{p / 2+1} a \tau^{p}}{(p-a)\left(2^{p / 2}-2^{p / a}\right)} h^{1-a / 2}\right]
\end{aligned}
$$

where

$$
K_{n}:=\sum_{n / d_{n} \leq 2^{j} \leq n / h} 2^{-(p / a-1) j} .
$$

This completes the proof of (3.23) since $K_{n} \rightarrow 0$ as $n \rightarrow \infty, n \in I$, since $d_{n} / n \rightarrow 0$.

\subsection{Proof of Theorem 2.4}

We proceed as in the proof of Th.2.3 by proving the convergence (2.11) for $X \in \mathrm{RV}_{a}$ and a subsequence indexed by $I$ verifying (3.21). Again, $a_{n} \sim \tau n^{1 / a}$ when $n \rightarrow \infty, n \in I$. Throughout the proof, $n$ belongs to $I$.

Choose $d_{n}=c n^{\kappa}$ for $n \geq n_{0}$, with $\kappa \in\left(\frac{2(a-2-\delta)}{(2+\delta)(a-2)}, 1\right)$ and $0<\delta<a-2$ if $a \leq 3$, $\delta=1$ if $a>3$. Then $d_{n} \rightarrow \infty, d_{n} / n \rightarrow 0$ as $n \rightarrow \infty, n \in I$ and (2.8) is satisfied. For $1<h<d_{n}<n$, recalling the notation (2.1), $T_{n}\left(\gamma_{a}\right)$ can be expressed as

$$
T_{n}\left(\gamma_{a}\right)=\max \left\{T_{n}^{0, h}, T_{n}^{h, d_{n}}, T_{n}^{d_{n}, n}\right\} .
$$

We will use the abbreviations

$$
T_{n}^{\prime}=T_{n}^{0, h}, \quad T_{n}^{\prime \prime}=T_{n}^{d_{n}, n} .
$$

With our choice of $d_{n},(3.22)$ is satisfied, see the proof of Th.2.3, hence for any $\varepsilon>0$,

$$
\lim _{h \rightarrow \infty} \limsup _{n \rightarrow \infty, n \in I} P\left(n^{-1 / a} T_{n}^{h, d_{n}}>\varepsilon\right)=0 .
$$

This will enable us to show that $n^{-1 / a} T_{n}\left(\gamma_{a}\right)$ and $n^{-1 / a} \max \left\{T_{n}^{\prime}, T_{n}^{\prime \prime}\right\}$ have the same limiting distribution. For the moment, we just note for ulterior use the related inequalities (3.25) and (3.26) below. First we notice that for any $r>0$,

$$
P\left(n^{-1 / a} T_{n}\left(\gamma_{a}\right) \leq r\right)=P\left(n^{-1 / a} \max \left\{T_{n}^{\prime}, T_{n}^{\prime \prime}, T_{n}^{h, d_{n}}\right\} \leq r\right) \leq P\left(n^{-1 / a} \max \left\{T_{n}^{\prime}, T_{n}^{\prime \prime}\right\} \leq r\right) .
$$


For a lower bound, we write

$$
\begin{aligned}
P\left(n^{-1 / a} \max \left\{T_{n}^{\prime}, T_{n}^{\prime \prime}\right\} \leq r-\varepsilon\right)= & P\left(n^{-1 / a} \max \left\{T_{n}^{\prime}, T_{n}^{\prime \prime}\right\} \leq r-\varepsilon, n^{-1 / a} T_{n}^{h, d_{n}} \leq \varepsilon\right) \\
& +P\left(n^{-1 / a} \max \left\{T_{n}^{\prime}, T_{n}^{\prime \prime}\right\} \leq r-\varepsilon, n^{-1 / a} T_{n}^{h, d_{n}}>\varepsilon\right) \\
\leq & P\left(n^{-1 / a} \max \left\{T_{n}^{\prime}, T_{n}^{\prime \prime}\right\} \leq r-n^{-1 / a} T_{n}^{h, d_{n}}\right) \\
& +P\left(n^{-1 / a} T_{n}^{h, d_{n}}>\varepsilon\right) \\
= & P\left(n^{-1 / a} \max \left\{T_{n}^{\prime}, T_{n}^{\prime \prime}\right\}+n^{-1 / a} T_{n}^{h, d_{n}} \leq r\right) \\
& +P\left(n^{-1 / a} T_{n}^{h, d_{n}}>\varepsilon\right) \\
\leq & P\left(n^{-1 / a} \max \left\{T_{n}^{\prime}, T_{n}^{\prime \prime}, T_{n}^{h, d_{n}}\right\} \leq r\right)+P\left(n^{-1 / a} T_{n}^{h, d_{n}}>\varepsilon\right),
\end{aligned}
$$

whence for any $r>0, \varepsilon>0$,

$$
P\left(n^{-1 / a} \max \left\{T_{n}^{\prime}, T_{n}^{\prime \prime}\right\} \leq r-\varepsilon\right)-P\left(n^{-1 / a} T_{n}^{h, d_{n}}>\varepsilon\right) \leq P\left(n^{-1 / a} T_{n}\left(\gamma_{a}\right) \leq r\right) .
$$

Now we analyse $\max \left\{T_{n}^{\prime}, T_{n}^{\prime \prime}\right\}$. To this aim, introducing the random vectors of $\mathbb{R}^{h}$

$$
U_{k}=U_{k, h}:=\left(S_{k-1,1}, \ldots, S_{k-1, h}\right)=\left(X_{k}, X_{k}+X_{k+1}, \ldots \ldots, X_{k}+\cdots+X_{k+h-1}\right),
$$

we consider the random measure $N_{n}$ on (the Borel $\sigma$-field of) $\mathbb{R}^{h}$ defined by

$$
N_{n}:=\sum_{k=1}^{n-h+1} \delta_{n^{-1 / a} U_{k}}
$$

where $\delta_{y}$ denotes the Dirac mass at the point $y$ of $\mathbb{R}^{h}$.

Write for $r>0$,

$$
B_{r}=B_{r, a}=\left\{\left(s_{1}, \ldots, s_{h}\right) \in \mathbb{R}^{h}: i^{-\gamma_{a}}\left|s_{i}\right| \leq r, i=1, \ldots, h\right\} .
$$

Then

$$
\left\{N_{n}\left(B_{r}^{c}\right)=0\right\}=\left\{n^{-1 / a} T_{n}^{\prime} \leq r\right\} .
$$

Indeed, $N_{n}\left(B_{r}^{c}\right)=0$, if and only if for each $k=1, \ldots, n, \delta_{n^{-1 / a} U_{k}}\left(B_{r}^{c}\right)=0$ or equivalently $n^{-1 / a}\left(X_{k}, \ldots, X_{k}+\cdots+X_{k+h-1}\right) \in B_{r}$. This means that for each $k=1, \ldots, n$, $n^{-1 / a} i^{-\gamma_{a}}\left|X_{k}+\cdots+X_{k+i}\right| \leq r$ for $i=1, \ldots, h$. Summing up, $N_{n}\left(B_{r}^{c}\right)=0$ if and only if

$$
n^{-1 / a} i^{-\gamma_{a}}\left|X_{k}+\cdots+X_{k+i-1}\right| \leq r, \quad i=1, \ldots, h, \quad k=1, \ldots, n-h+1
$$

or if and only if $n^{-1 / a} T_{n}^{\prime} \leq r$.

Introducing the interval of integers $\llbracket u, v \rrbracket:=[u, v] \cap \mathbb{N}$, with the usual convention $[u, v]=\emptyset$ when $v<u$, consider for $j=1, \ldots, n$ the sets

$$
\begin{aligned}
I_{j}=I(j, h) & :=\llbracket j-h, j+h \rrbracket \cap \llbracket 1, n-h+1 \rrbracket \\
I_{j}^{c} & :=\llbracket 1, n-h+1 \rrbracket \backslash I_{j}=\llbracket 1, j-h-1 \rrbracket \cup \llbracket j+h+1, n-h+1 \rrbracket .
\end{aligned}
$$

Now define

$$
N_{n}^{(j)}=\sum_{k \in I_{j}^{c}} \delta_{n^{-1 / a} U_{k}}
$$

In what follows we use the functions $f_{r, \varepsilon}$ and the random vectors $X_{n, i}, Y_{n, i}, Z_{n, i}$ introduced in the proof of Th. 2.2. We have

$$
\begin{aligned}
P\left(n^{-1 / a} \max \left\{T_{n}^{\prime}, T_{n}^{\prime \prime}\right\} \leq r\right) & =P\left(n^{-1 / a} T_{n}^{\prime} \leq r, n^{-1 / a} T_{n}^{\prime \prime} \leq r\right) \\
& =\mathrm{E}\left(\mathbb{1}\left\{N_{n}\left(B_{r}^{c}\right)=0\right\} \mathbb{1}\left\{n^{-1 / a} T_{n}^{\prime \prime} \leq r\right\}\right) \\
& \leq \mathrm{E}\left(\mathbb{1}\left\{N_{n}\left(B_{r}^{c}\right)=0\right\} f_{r, \varepsilon}\left(\sum_{i=1}^{n} X_{n, i}\right)\right) .
\end{aligned}
$$


Recalling (3.2), we continue the above estimation by

$$
\begin{aligned}
P\left(n^{-1 / a} \max \left\{T_{n}^{\prime}, T_{n}^{\prime \prime}\right\} \leq r\right) \leq & \sum_{j=1}^{n} \mathrm{E} \mathbb{1}\left\{N_{n}\left(B_{r}^{c}\right)=0\right\}\left[f_{r, \varepsilon}\left(Z_{n, j}+X_{n, j}\right)-f_{r, \varepsilon}\left(Z_{n, j}+Y_{n, j}\right)\right] \\
& +\mathrm{E} \mathbb{1}\left\{N_{n}\left(B_{r}^{c}\right)=0\right\} f_{r, \varepsilon}\left(\sum_{i=1}^{n} Y_{n, i}\right) \\
= & P_{n 1}(r, \varepsilon)+P_{n 2}(r, \varepsilon)+P_{n 3}(r, \varepsilon)
\end{aligned}
$$

where

$$
\begin{gathered}
P_{n 1}(r, \varepsilon)=\sum_{j=1}^{n} \mathrm{E} \mathbb{1}\left\{N_{n}^{(j)}\left(B_{r}^{c}\right)=0\right\}\left[f\left(Z_{n, j}+X_{n, j}\right)-f\left(Z_{n, j}+Y_{n, j}\right)\right], \\
P_{n 2}(r, \varepsilon)=\sum_{j=1}^{n} \mathrm{E}\left[\mathbb{1}\left\{N_{n}\left(B_{r}^{c}\right)=0\right\}-\mathbb{1}\left\{N_{n}^{(j)}\left(B_{r}^{c}\right)=0\right\}\right]\left[f\left(Z_{n, j}+X_{n, j}\right)-f\left(Z_{n, j}+Y_{n, j}\right)\right],
\end{gathered}
$$

and

$$
P_{n 3}(r, \varepsilon)=\mathrm{E} \mathbb{1}\left\{N_{n}\left(B_{r}^{c}\right)=0\right\} f_{r, \varepsilon}\left(\sum_{j=1}^{n} Y_{n, j}\right) .
$$

To estimate $P_{n 1}$ we use Taylor's expansion which gives

$$
\begin{array}{r}
P_{n 1}(r, \varepsilon)=\sum_{j=1}^{n} \mathrm{E}\left(\mathbb { 1 } \{ N _ { n } ^ { ( j ) } ( B _ { r } ^ { c } ) = 0 \} \left[f^{\prime}\left(Z_{n, j}\right)\left(X_{n, j}\right)+f^{\prime \prime}\left(Z_{n, j}\left(X_{n, j}\right)^{2}+R_{1}(j)\right.\right.\right. \\
\left.\left.-f^{\prime}\left(Z_{n, j}\right)\left(Y_{n, j}\right)-f^{\prime \prime}\left(Z_{n, j}\right)\left(Y_{n, j}\right)^{2}-R_{2}(j)\right]\right),
\end{array}
$$

where

$$
\begin{aligned}
\left|R_{m}(j)\right| & \leq 6^{-\delta}\left\|f_{r, \varepsilon}^{\prime \prime}\right\|^{1-\delta}\left\|f_{r, \varepsilon}^{\prime \prime \prime}\right\|^{\delta}\left(\left\|X_{n, j}\right\|_{\infty}^{2+\delta}+\left\|Y_{n, j}\right\|_{\infty}^{2+\delta}\right) \\
& \leq C_{\delta}^{\prime} \varepsilon^{-2-\delta} d_{n}^{-\gamma_{a}} n^{-(2+\delta) / a}\left(\left|X_{j}\right|^{2+\delta}+\left|Y_{j}\right|^{2+\delta}\right), \quad m=1,2
\end{aligned}
$$

As $\mathbb{1}\left\{N_{n}^{(j)}\left(B^{c}(x)\right)=0\right\}$ and $X_{n, j}$ are independent, $\mathbb{1}\left\{N_{n}^{(j)}\left(B^{c}(r)\right)=0\right\} f^{\prime}\left(Z_{n, j}\right)$ and $\mathbb{1}\left\{N_{n}^{(j)}\left(B^{c}(r)\right)=0\right\} f^{\prime \prime}\left(Z_{n, j}\right)$ are respectively a random linear form on $\mathbb{R}^{k_{n}}$ and a random bilinear symetric form on $\mathbb{R}^{k} \times \mathbb{R}^{k}$, both independent of $X_{n, j}$. By lemmas A.2 and A.3 in Appendix, recalling that $X_{n, j}$ and $Y_{n, j}$ have the same (null) expectation and covariance matrix, one sees that

$$
\begin{gathered}
\mathrm{E}\left(\mathbb{1}\left\{N_{n}^{(j)}\left(B^{c}(r)\right)=0\right\}\left[f^{\prime}\left(Z_{n, j}\right)\left(X_{n, j}\right)+f^{\prime \prime}\left(Z_{n, j}\right)\left(X_{n, j}\right)^{2}\right]\right)= \\
\mathrm{E}\left(\mathbb{1}\left\{N_{n}^{(j)}\left(B^{c}(r)\right)=0\right\}\left[f^{\prime}\left(Z_{n, j}\right)\left(Y_{n, j}\right)+f^{\prime \prime}\left(Z_{n, j}\right)\left(Y_{n, j}\right)^{2}\right]\right) .
\end{gathered}
$$

This yields

$$
\begin{aligned}
\left|P_{n 1}(r, \varepsilon)\right| & \leq \sum_{j=1}^{n} \mathrm{E} \mathbb{1}\left\{N_{n}^{(j)}\left(B^{c}(x)\right)=0\right\}\left[\left|R_{1}(j)\right|+\left|R_{2}(j)\right|\right] \leq c_{\delta} \sum_{j=1}^{n} \mathrm{E}\left[\left|R_{1}(j)\right|+\left|R_{2}(j)\right|\right] \\
& \leq C_{\delta}^{\prime} \varepsilon^{-2-\delta} d_{n}^{-\gamma_{a}} n^{1-(2+\delta) / a}\left[\mathrm{E}\left|X_{1}\right|^{2+\delta}+\mathrm{E}\left|Y_{1}\right|^{2+\delta}\right]
\end{aligned}
$$

By our choice of $d_{n}$, we have for each $r>0$ and $\varepsilon>0$,

$$
\limsup _{n \rightarrow \infty, n \in I}\left|P_{n 1}(r, \varepsilon)\right|=0 .
$$


To estimate $P_{n 2}(r, \varepsilon)$, we introduce

$$
\tilde{N}_{n}^{(j)}=N_{n}-N_{n}^{(j)}=\sum_{k \in I(j, h)} \delta_{n^{-1 / a}\left(X_{k}, \ldots, X_{k}+\cdots+X_{k+h-1}\right)}
$$

and notice that $E:=\left\{N_{n}\left(B_{r}^{c}\right)=0\right\} \subset F:=\left\{N_{n}^{(j)}\left(B_{r}^{c}\right)=0\right\}$. Therefore

$$
\begin{aligned}
\mathbb{1}_{F}-\mathbb{1}_{E}=\mathbb{1}_{F \backslash E}=\mathbb{1}_{F \cap E^{c}} & =\mathbb{1}\left\{N_{n}^{(j)}\left(B_{r}^{c}\right)=0, N_{n}\left(B_{r}^{c}\right) \neq 0,\right\} \\
& =\mathbb{1}\left\{N_{n}^{(j)}\left(B_{r}^{c}\right)=0, \widetilde{N}_{n}^{(j)}\left(B_{r}^{c}\right) \neq 0\right\} \\
& \leq \mathbb{1}\left\{\widetilde{N}_{n}^{(j)}\left(B_{r}^{c}\right) \neq 0\right\} .
\end{aligned}
$$

Now, as $\left\|f_{r, \varepsilon}^{\prime}\right\|_{\infty} \leq c_{1} \varepsilon^{-1}$, the function $f_{r, \varepsilon}$ is Lipschitz with constant $c_{1} \varepsilon^{-1}$, that is $\left|f_{r, \varepsilon}(x)-f_{r, \varepsilon}(y)\right| \leq c_{1} \varepsilon^{-1}\|x-y\|_{\infty}$, whence

$$
\begin{aligned}
\left|P_{n 2}(r, \varepsilon)\right| & \leq \sum_{j=1}^{n} \mathrm{E} \mathbb{1}\left\{\tilde{N}_{n}^{(j)}\left(B_{r}^{c}\right) \neq 0\right\}\left|f\left(Z_{n, j}+X_{n, j}\right)-f\left(Z_{n, j}+Y_{n, j}\right)\right| \\
& \left.\leq c_{1} \varepsilon^{-1} \sum_{j=1}^{n} \mathrm{E} \mathbb{1}\left\{\tilde{N}_{n}^{(j)}\left(B_{r}^{c}\right) \neq 0\right\}\left[\left\|X_{n, j}\right\|_{\infty}+\| Y_{n, j}\right) \|_{\infty}\right] .
\end{aligned}
$$

Recalling (3.8), we get

$$
\left|P_{n 2}(r, \varepsilon)\right| \leq c_{1} \varepsilon^{-1} n^{-1 / a} d_{n}^{-\gamma_{a}}\left(P_{n 2}^{\prime}(r)+P_{n 2}^{\prime \prime}(r)\right),
$$

where

$$
P_{n 2}^{\prime}(r)=\sum_{j=1}^{n} \mathrm{E} \mathbb{1}\left\{\tilde{N}_{n}^{(j)}\left(B_{r}^{c}\right) \neq 0\right\}\left|X_{j}\right|, \quad P_{n 2}^{\prime \prime}(r)=\sum_{j=1}^{n} \mathrm{E} \mathbb{1}\left\{\widetilde{N}_{n}^{(j)}\left(B_{r}^{c}\right) \neq 0\right\}\left|Y_{j}\right| .
$$

As $Y_{j}$ and $\widetilde{N}_{n}^{(j)}$ are independent,

$$
P_{n 2}^{\prime \prime}(r)=\sum_{j=1}^{n} P\left(\tilde{N}_{n}^{(j)}\left(B_{r}^{c}\right)\right) \mathrm{E}\left|Y_{j}\right|
$$

Since

we see that

$$
\left\|\left(X_{k}, \ldots, X_{k}+\cdots+X_{k+h-1}\right)\right\|_{\infty} \leq h \max _{k \leq i \leq k+h-1}\left|X_{i}\right|,
$$

$$
\begin{aligned}
\left\{\widetilde{N}_{n}^{(j)}\left(B_{r}^{c}\right) \neq 0\right\} & \subset \bigcup_{k \in I(j, h)}\left\{n^{-1 / a}\left(X_{k}, \ldots, X_{k}+\cdots+X_{k+h-1}\right) \in B_{r}^{c}\right\} \\
& =\bigcup_{k \in I(j, h)}\left\{n^{-1 / a}\left\|\left(X_{k}, \ldots, X_{k}+\cdots+X_{k+h-1}\right)\right\|_{\infty}>r\right\} \\
& \subset \bigcup_{k \in I(j, h)}\left\{\max _{k \leq i \leq k+h-1}\left|X_{i}\right|>r n^{1 / a} h^{-1}\right\} \\
& =\bigcup_{k \in I(j, h)} \bigcup_{i=k}^{k+h-1}\left\{\left|X_{i}\right|>r n^{1 / a} h^{-1}\right\} .
\end{aligned}
$$

This yields

$$
\begin{aligned}
P_{n 2}^{\prime \prime}(r) & \leq \sum_{j=1}^{n} \sum_{k \in I(j, h)} \sum_{i=k}^{k+h-1} P\left(\left|X_{i}\right|>r n^{1 / a} h^{-1}\right) \mathrm{E}\left|Y_{j}\right| \\
& \leq n(2 h) h P\left(\left|X_{1}\right|>r n^{1 / a} h^{-1}\right) \mathrm{E}\left|Y_{1}\right| \\
& \leq 3 \tau^{a} \mathrm{E}\left|Y_{1}\right| h^{2+a} r^{-a},
\end{aligned}
$$


for $n \geq n_{0}(h, r, L), n \in I$, using (3.21) in the last step.

Similarly we estimate

$$
\begin{aligned}
P_{n 2}^{\prime}(r) & \leq \sum_{j=1}^{n} \sum_{k \in I(j, h)} \sum_{i=k}^{k+h-1} \mathrm{E} \mathbb{1}\left\{\left|X_{i}\right|>r n^{1 / a} h^{-1}\right\}\left|X_{j}\right| \\
& \leq 2 h^{2} n\left[\mathrm{E} \mathbb{1}\left\{\left|X_{2}\right|>r n^{1 / a} h^{-1}\right\}\left|X_{1}\right|+\mathrm{E} \mathbb{1}\left\{\left|X_{1}\right|>r n^{1 / a} h^{-1}\right\}\left|X_{1}\right|\right] \\
& =2 h^{2} n\left[P\left(\left|X_{2}\right|>r n^{1 / a} h^{-1}\right) \mathrm{E}\left|X_{1}\right|+\mathrm{E} \mathbb{1}\left\{\left|X_{1}\right|>r n^{1 / a} h^{-1}\right\}\left|X_{1}\right|\right] \\
& \leq 3 \tau^{a}\left(2 \mathrm{E}\left|X_{1}\right| r^{-a}+a r^{1-a}\right) h^{2+a} n^{1 / a}
\end{aligned}
$$

recalling that $a>2$ and using (i) of Lemma 3.2 with $c_{n}=\tau n^{1 / a}, n \in I$. Now (3.29), (3.30) and (3.31) yield

$$
\limsup _{n \rightarrow \infty, n \in I}\left|P_{n 2}(r, \varepsilon)\right|=0 .
$$

Since the sequences $\left(X_{k}\right)_{k \geq 1}$ and $\left(Y_{k}\right)_{k \geq 1}$ are independent,

$$
P_{n 3}(r, \varepsilon)=P\left(N_{n}\left(B_{r}^{c}\right)=0\right) \mathrm{E} f_{r, \varepsilon}\left(\sum_{i=1}^{n} Y_{n, i}\right)
$$

hence recalling that our choice of $d_{n}$ enables us to apply the versions of Cor.2.2 and Th.2.3 for the subsequence indexed by $I$ verifying (3.21), we obtain

$$
\limsup _{n \rightarrow \infty, n \in I} P_{n 3}(r, \varepsilon)=P\left(\tau Y_{a} \leq r\right) P\left(\sigma T\left(\gamma_{a}\right) \leq r+\varepsilon\right)
$$

Collecting (3.27), (3.28), (3.32) and (3.33), we find for any $r>0$ and $\varepsilon>0$,

$$
\limsup _{n \rightarrow \infty, n \in I} P\left(n^{-1 / a} \max \left\{T_{n}^{\prime}, T_{n}^{\prime \prime}\right\} \leq r\right) \leq P\left(\tau Y_{a} \leq r\right) P\left(\sigma T\left(\gamma_{a}\right) \leq r+\varepsilon\right)
$$

Accounting (3.25), this implies for any $r>0$ and $\varepsilon>0$,

$$
\limsup _{n \rightarrow \infty, n \in I} P\left(n^{-1 / a} T_{n}^{\left(\gamma_{a}\right)} \leq r\right) \leq P\left(\tau Y_{a} \leq r\right) P\left(\sigma T\left(\gamma_{a}\right) \leq r+\varepsilon\right)
$$

In a similar way we prove for any $r>0$ and $0<\varepsilon<r / 2$,

$$
\liminf _{n \rightarrow \infty, n \in I} P\left(n^{-1 / a} \max \left\{T_{n}^{\prime}, T_{n}^{\prime \prime}\right\} \leq r-\varepsilon\right) \geq P\left(\tau Y_{a} \leq r-\varepsilon\right) P\left(\sigma T\left(\gamma_{a}\right) \leq r-2 \varepsilon\right) .
$$

Accounting (3.26), this implies for any $h>1, r>0$ and $0<\varepsilon<r / 2$,

$$
\begin{aligned}
\liminf _{n \rightarrow \infty, n \in I} P\left(n^{-1 / a} T_{n}\left(\gamma_{a}\right) \leq r\right) \geq P & \left(\tau Y_{a} \leq r-\varepsilon\right) P\left(\sigma T\left(\gamma_{a}\right) \leq r-2 \varepsilon\right) \\
& -\limsup _{n \rightarrow \infty, n \in I} P\left(n^{-1 / a} T_{n}^{h, d_{n}}>\varepsilon\right) .
\end{aligned}
$$

In this inequality, only the limsup term depends on $h$. So letting $h$ tend to infinity we obtain by (3.24)

$$
\liminf _{n \rightarrow \infty, n \in I} P\left(n^{-1 / a} T_{n}\left(\gamma_{a}\right) \leq r\right) \geq P\left(\tau Y_{a} \leq r-\varepsilon\right) P\left(\sigma T\left(\gamma_{a}\right) \leq r-2 \varepsilon\right)
$$

Finally, gathering (3.34) and (3.35) and using the continuity of the distributions functions of $Y_{a}$ and $T\left(\gamma_{a}\right)$, we complete the proof by letting $\varepsilon \rightarrow 0$. 


\subsection{Proof of Theorem 2.5}

As a preliminary, we construct a sequence of reals $\left(m_{i}\right)_{i \geq 0}$ increasing to infinity and a slowly varying function $L=L_{a}$ such that

$\alpha$ ) for every $a>2, x^{-a} L_{a}(x)$ decreases from 1 to 0 on $\left[m_{0}, \infty\right)$;

B) $L_{a}\left(m_{2 i}\right)_{i \geq 1}$ increases to infinity and $L_{a}\left(m_{2 i+1}\right)_{i \geq 0}$ decreases to zero.

We start with an arbitrary sequence $\left(m_{i}\right) \uparrow \infty$ on which we will progressively put some constraints. Choosing $m_{0} \geq \mathrm{e}^{1 / 2}$, we define the function $\mathfrak{i}:\left[m_{0}, \infty\right) \rightarrow \mathbb{N}$ by $\mathfrak{i}:=\mathbb{1}_{\left\{m_{0}\right\}}+\sum_{i=1}^{\infty} i \mathbb{1}_{\left(m_{i-1}, m_{i}\right]}$, that is

$$
\mathfrak{i}\left(m_{0}\right)=1, \quad \mathfrak{i}(u)=i \quad \text { if } m_{i-1}<u \leq m_{i}, \quad i \geq 1 .
$$

Now we define $L_{a}$ on $\left[m_{0}, \infty\right)$ by

$$
L_{a}(x):=m_{0}^{a} \exp \left(\int_{m_{0}}^{x} \frac{(-1)^{\mathfrak{i}(u)}}{\log u} \frac{\mathrm{d} u}{u}\right) .
$$

By A.4-Th.A.11 below, $L$ is clearly a slowly varying function. We can already check $\alpha$ ) without additional conditions on $\left(m_{i}\right)_{i \geq 1}$. Obviously $m_{0}^{-a} L_{a}\left(m_{0}\right)=1$. Writing

$$
x^{-a}=m_{0}^{-a} \exp \left(\int_{m_{0}}^{x}-a \frac{\mathrm{d} u}{u}\right), \quad x \geq m_{0},
$$

gives

$$
x^{-a} L_{a}(x)=\exp \left(\int_{m_{0}}^{x}\left(-a+\frac{(-1)^{\mathfrak{i}(u)}}{\log u}\right) \frac{\mathrm{d} u}{u}\right) .
$$

For $u \geq \mathrm{e}^{1 / 2},-a+(-1)^{\mathfrak{i}(u)} / \log u \leq-a+2<0$ which implies the decreasingness of $x^{-a} L_{a}(x)$ on $\left[m_{0}, \infty\right)$ and its convergence to 0 at infinity.

To find conditions on $m_{i}$ implying $\beta$ ), we note that for $i \geq 2$,

$$
\begin{aligned}
L_{a}\left(m_{i}\right) & =m_{0}^{a} \exp \left(\int_{m_{0}}^{m_{i-2}} \frac{(-1)^{\mathrm{i}(u)}}{\log u} \frac{\mathrm{d} u}{u}+\int_{m_{i-2}}^{m_{i-1}} \frac{(-1)^{\mathrm{i}(u)}}{\log u} \frac{\mathrm{d} u}{u}+\int_{m_{i-1}}^{m_{i}} \frac{(-1)^{\mathrm{i}(u)}}{\log u} \frac{\mathrm{d} u}{u}\right) \\
& =L_{a}\left(m_{i-2}\right) \exp \left((-1)^{i-1} \log \left(\frac{\log m_{i-1}}{\log m_{i-2}}\right)+(-1)^{i}\left(\frac{\log m_{i}}{\log m_{i-1}}\right)\right) .
\end{aligned}
$$

Therefore

$$
\frac{L_{a}\left(m_{i}\right)}{L_{a}\left(m_{i-2}\right)}= \begin{cases}\frac{\log m_{i-2} \log m_{i}}{\left(\log m_{i-1}\right)^{2}} & \text { if } i \text { is even, } \\ \frac{\left(\log m_{i-1}\right)^{2}}{\log m_{i-2} \log m_{i}} & \text { if } i \text { is odd. }\end{cases}
$$

Hence the increasingness of $L_{a}\left(m_{2 i}\right)_{i \geq 1}$ as well as the decreasingness of $L_{a}\left(m_{2 i+1}\right)_{i \geq 0}$ require that $\left(m_{i}\right)_{i \geq 2}$ satisfies the condition

$$
\frac{\log m_{i-2} \log m_{i}}{\left(\log m_{i-1}\right)^{2}}>1 \text {. }
$$

This means that the sequence $\left(\log \log m_{i}\right)_{i \geq 0}$ has to be strictly convex. A simple choice satisfying this condition is $\log \log m_{i}=i^{b}$, with $b>1$. In particular with $b=2, m_{i}=$ $\exp \left(\exp \left(i^{2}\right)\right)$, since $i^{2}+(i-2)^{2}-2(i-1)^{2}=2$, we find

$$
\frac{\log m_{i-2} \log m_{i}}{\left(\log m_{i-1}\right)^{2}}=\mathrm{e}^{2} \text {. }
$$


In this case, $L_{a}\left(m_{2 i}\right) \uparrow \infty, L_{a}\left(m_{2 i+1}\right) \downarrow 0$. The same holds (with less explicit formulas) for $b \neq 2$ since $i^{b}+(i-2)^{b}-2(i-1)^{b}=b(b-1)+o(1)$. From now on, we fix $m_{i}:=\exp \left(\exp \left(i^{2}\right)\right)$, $i \geq 0$.

Next, we choose the distribution of $X$ symmetric such that

$$
P(|X|>x)= \begin{cases}1 & \text { if } x<\mathrm{e} \\ x^{-a} L_{a}(x) & \text { if } x \geq \mathrm{e} .\end{cases}
$$

Clearly, $X \in \mathrm{RV}_{a} \backslash \bigcup_{\tau \in[0, \infty]} \mathrm{RV}^{(\tau)}, \mathrm{E} X=0$ and as $a>2, \sigma^{2}=\mathrm{E} X^{2}<\infty$.

To prove i), let us recall that $L_{a}\left(m_{2 i-1}\right) \rightarrow 0$ as $i \rightarrow \infty$. Now choose $n_{i}:=\left[m_{2 i-1}^{a}\right]$. It is clear that $n_{i}^{1 / a} \sim m_{2 i-1}$ and by A.4-Cor.A.10 ii), one checks that $L_{a}\left(n_{i}^{1 / a}\right) \sim L_{a}\left(m_{2 i-1}\right)$, whence $L_{a}\left(n_{i}^{1 / a}\right) \rightarrow 0$ as $i \rightarrow \infty$. Then the convergence (2.12) follows by a continuous mapping argument from A.2-Th.A.4.

To prove ii), we can build the sequence $n_{i}=n_{i}(a, \tau)$ as follows. First we note that for $t \in\left[m_{2 i-1}, m_{2 i}\right]$,

$$
L_{a}(t)=L_{a}\left(m_{2 i-1}\right) \exp \left(\int_{m_{2 i-1}}^{t} \frac{1}{\log u} \frac{\mathrm{d} u}{u}\right)
$$

whence

$$
L_{a}(t)=\frac{L_{a}\left(m_{2 i-1}\right)}{\log m_{2 i-1}} \log t, \quad t \in\left[m_{2 i-1}, m_{2 i}\right] .
$$

Recalling that $m_{i}=\exp \left(\exp \left(i^{2}\right)\right)$, we note also that

$$
\frac{m_{2 i}}{m_{2 i-1}^{a}}=\exp \left(\exp \left(4 i^{2}\right)(1-a \exp (-4 i+1))\right)>1, \quad i>\frac{1+\log a}{4} .
$$

So for $i>(1+\log a) / 4, m_{2 i-1}<m_{2 i-1}^{a}<m_{2 i}$, whence by (3.36),

$$
L_{a}\left(m_{2 i-1}^{a}\right)=\frac{L_{a}\left(m_{2 i-1}\right)}{\log m_{2 i-1}} \log \left(m_{2 i-1}^{a}\right)=a L_{a}\left(m_{2 i-1}\right) .
$$

Therefore $L_{a}\left(m_{2 i-1}^{a}\right)$ tends to 0 as $i$ tends to infinity. As moreover $L_{a}\left(m_{2 i}\right)$ tends to infinity, $L_{a}\left(m_{2 i-1}^{a}\right)<\tau^{a}<L_{a}\left(m_{2 i}\right)$ for $i$ large enough. Then by increasingness and continuity of $L_{a}$ in $\left[m_{2 i-1}^{a}, m_{2 i}\right]$ there is a unique $t_{i}$ in $\left(m_{2 i-1}^{a}, m_{2 i}\right)$ such that $L_{a}\left(t_{i}\right)=a \tau^{a}$. Furthermore, since $t_{i}>m_{2 i-1}^{a}, t_{i}^{1 / a} \in\left[m_{2 i-1}, m_{2 i}\right]$, so by (3.36), $L_{a}\left(t_{i}^{1 / a}\right)=\tau^{a}$. Now puting $n_{i}:=\left[t_{i}\right]$, we get by A.4-Cor.A.10 ii) that $\lim _{i \rightarrow \infty} L_{a}\left(n_{i}^{1 / a}\right)=\tau^{a}$ that is $L_{a}$ satisfies (3.21). Then we can apply the subsequence version established in the proof of Th. 2.4 which gives the convergence in distribution of $n_{i}^{-1 / a} T_{n_{i}}\left(\gamma_{a}\right)$ to $\max \left\{\sigma T\left(\gamma_{a}\right), \tau Y_{a},\right\}$.

To prove iii), we choose $n_{i}=\left[m_{2 i}\right]$. Let us first recall that by [12, Th.10], the sequence $\left(n^{-1 / a} T_{n}\left(\gamma_{a}\right)\right)_{n \geq 1}$ is stochastically bounded if and only if $\sup _{t>0} t^{a} P(|X|>t)$ is finite. We will adapt the argument given in the proof to prove that $\left(n_{i}^{-1 / a} T_{n_{i}}\left(\gamma_{a}\right)\right)_{i \geq 0}$ is not stochastically bounded. Beforehand, we recall that for $i>(1+\log a) / 4, m_{2 i}^{1 / a}>m_{2 i-1}$, so by (3.36), $L_{a}\left(m_{2 i}^{1 / a}\right)=a^{-1} L_{a}\left(m_{2 i}\right)$. This together with the slow variation of $L_{a}$ and A.4-Cor.A.10 ii) gives for every $r>0$

$$
L_{a}\left(r n_{i}^{1 / a}\right) \sim L_{a}\left(n_{i}^{1 / a}\right) \sim L_{a}\left(m_{2 i}^{1 / a}\right)=a^{-1} L_{a}\left(m_{2 i}\right) \underset{i \rightarrow \infty}{\longrightarrow} \infty .
$$

Now assume that $\left.\left(n_{i}^{-1 / a} T_{n_{i}}\left(\gamma_{a}\right)\right)\right)_{i \geq 0}$ is stochastically bounded. This implies the stochastic boundedness of $\left(n_{i}^{-1 / a} \max _{1 \leq k \leq n_{i}}\left|X_{k}\right|\right)_{i \geq 0}$, that is

$$
\lim _{r \rightarrow \infty} \sup _{i \geq 0} P\left(n_{i}^{-1 / a} \max _{1 \leq k \leq n_{i}}\left|X_{k}\right|>r\right)=0 .
$$


By independence and identical distribution of the $X_{k}$ 's and (3.38), for every $r>0$,

$$
\begin{aligned}
P\left(n_{i}^{-1 / a} \max _{1 \leq k \leq n_{i}}\left|X_{k}\right|>r\right)=1-\left(1-\frac{L_{a}\left(r n_{i}^{1 / a}\right)}{r^{a} n_{i}}\right)^{n_{i}} & \\
& =1-(1+o(1)) \exp \left(\frac{-L_{a}\left(r n_{i}^{1 / a}\right)}{r^{a}}\right) \underset{i \rightarrow \infty}{\longrightarrow} 1,
\end{aligned}
$$

whence

$$
\sup _{i \geq 0} P\left(n_{i}^{-1 / a} \max _{1 \leq k \leq n_{i}}\left|X_{k}\right|>r\right)=1, \quad r>0
$$

which contradicts (3.39). Hence $\left(n_{i}^{-1 / a} T_{n_{i}}\left(\gamma_{a}\right)\right)_{i \geq 0}$ is not stochastically bounded.

Now let us turn on the proof of the convergence of $a_{n_{i}}^{-1} T_{n_{i}}\left(\gamma_{a}\right)$. By A.4-Cor.A.10 ii), $L_{a}\left(n_{i}\right) \sim L_{a}\left(m_{2 i}\right)$ which tends to infinity. In [12], Th. 5b) states that if $X \in \mathrm{RV}_{a}(\infty)$, $a_{n}^{-1} T_{n}\left(\gamma_{a}\right)$ converges in distribution to $Y_{a}$. So we have only to check the adaptation of this theorem for the subsequence indexed by $n_{i}$ under the weaker assumption $L_{a}\left(n_{i}\right) \rightarrow \infty$ instead of $L_{a}(x) \rightarrow \infty$ as $x \rightarrow \infty$. In fact in the original proof of [12, Th. 5b)], including the 3 premiminary lemmas, everything is writen under the more general assumption $X \in \mathrm{RV}_{a}$, except the last paragraph starting by "We see that when $a>2$, (35) and (36) are valid provided that $\lim _{n \rightarrow \infty} a_{n}^{-1} n^{1 / a}=0 \ldots$.. Therefore everything works when substituting $n$ by $n_{i}$ until this last paragraph and it remains only to justify the convergence $\lim _{i \rightarrow \infty} a_{n_{i}}^{-1} n_{i}^{1 / a}=0$.

By (3.37), $m_{2 i}^{1 / a} / m_{2 i-1} \rightarrow \infty$ whence $n_{i}^{1 / a} \in\left(m_{2 i-1}, m_{2 i}\right)$ for $i$ large enough and $L_{a}\left(n_{i}^{1 / a}\right)=\frac{1}{a} L_{a}\left(n_{i}\right)$. Hence

$$
n_{i} P\left(|X|>n_{i}^{1 / a}\right)=\frac{1}{a} L_{a}\left(n_{i}\right) \underset{i \rightarrow \infty}{\longrightarrow} \infty .
$$

On the other hand, as $x^{-a} L_{a}(x)$ is continuous and decreasing on $\left[m_{0}, \infty\right)$,

$$
n_{i} P\left(|X|>a_{n_{i}}\right)=1
$$

Therefore for $i$ large enough $P\left(|X|>n_{i}^{1 / a}\right)>P\left(|X|>a_{n_{i}}\right)$, whence $n_{i}^{1 / a}<a_{n_{i}}$. In particular,

$$
\lambda:=\limsup \frac{n_{i}^{1 / a}}{a_{n_{i}}} \leq 1
$$

Suppose that $\lambda$ is positive. Then there exists a subsequence $\left(n_{i_{j}}\right)$ of $\left(n_{i}\right)$ such that $n_{i_{j}}^{1 / a} / a_{n_{i_{j}}}>\lambda / 2$, whence

$$
1=n_{i_{j}} P\left(|X|>a_{n_{i_{j}}}\right)>n_{i_{j}} P\left(|X|>\frac{2}{\lambda} n_{i_{j}}^{1 / a}\right) \sim \frac{\lambda^{a}}{a 2^{a}} L_{a}\left(m_{2 i_{j}}\right) \underset{j \rightarrow \infty}{\longrightarrow} \infty,
$$

which is contradictory. Therefore $\lambda=0$ and the proof of iii) is complete.

\subsection{Proof of Theorem 2.6}

Assume that $L(x)$ as no limit as $x \rightarrow \infty$. Then

$$
\theta^{a}:=\liminf _{x \rightarrow \infty} L(x)<{\theta^{\prime}}^{a}:=\limsup _{x \rightarrow \infty} L(x)
$$

and there are two sequences of reals $\left(t_{i}\right)_{i \geq 1}$ and $\left(t_{i}^{\prime}\right)_{i \geq 1}$ increasing to infinity such that

$$
L\left(t_{i}\right) \underset{i \rightarrow \infty}{\longrightarrow} \theta^{a}, \quad L\left(t_{i}^{\prime}\right) \underset{i \rightarrow \infty}{\longrightarrow} \theta^{\prime a} .
$$


Now puting $n_{i}:=\left[t_{i}^{a}\right]$, we note that $n_{i}^{1 / a} \sim t_{i}$ and by A.4-Cor.A.10 ii),

$$
L\left(n_{i}^{1 / a}\right) \sim L\left(t_{i}\right) \underset{i \rightarrow \infty}{\longrightarrow} \theta^{a} .
$$

In the case where $\theta=0$, then $n_{i}^{-1 / a} T_{n_{i}}\left(\gamma_{a}\right)$ converges in distribution to $\sigma T\left(\gamma_{a}\right)$ by Th.A.4 and continuous mapping. If $0<\theta<\infty$, as (3.21) is satisfied by $\left(n_{i}\right)_{i \geq 1}$, the corresponding subsequence version of Th.2.4 gives the weak convergence of $n_{i}^{-1 / a} T_{n_{i}}\left(\gamma_{a}\right)$ to $V_{a, \sigma, \theta}$. When $\theta^{\prime}<\infty$, the same argument applied with $n_{i}^{\prime}:=\left[t_{i}^{\prime a}\right]$ gives the weak convergence of $n_{i}^{\prime-1 / a} T_{n_{i}^{\prime}}\left(\gamma_{a}\right)$ to $V_{a, \sigma, \theta^{\prime}}$. In the special case where $\theta^{\prime}=\infty$ we have to modify the definition of $n_{i}^{\prime}$ in the following way. As the quantile sequence $\left(a_{n}\right)_{n>1}$ is nondecreasing and tends to infinity, we set $n_{i}^{\prime}:=\max \left\{k \geq 1: a_{k} \leq t_{i}^{\prime}\right\}$. As $\left(t_{i}^{\prime}\right)_{i \geq 1}$ is increasing, $\left(a_{n_{i}^{\prime}}\right)_{i \geq 1}$ is nondecreasing and verifies $a_{n_{i}^{\prime}} \leq t_{i}^{\prime}<a_{1+n_{i}^{\prime}}$. As by Lemma $3.2 \mathrm{iv),} a_{n}=n^{1 / a} l(n)$ with $l$ slowly varying, this implies

$$
1 \leq \frac{t_{i}^{\prime}}{a_{n_{i}^{\prime}}} \leq\left(\frac{1+n_{i}^{\prime}}{n_{i}^{\prime}}\right)^{1 / a} \frac{l\left(1+n_{i}^{\prime}\right)}{l\left(n_{i}^{\prime}\right)} \underset{i \rightarrow \infty}{\longrightarrow} 1,
$$

so $a_{n_{i}^{\prime}} \sim t_{i}^{\prime}$ and $L\left(a_{n_{i}^{\prime}}\right) \rightarrow \infty$. To deduce from this that $a_{n_{i}^{\prime}}^{-1} T_{n_{i}^{\prime}}\left(\gamma_{a}\right)$ converges to $V_{a, \sigma, \infty}$ it remains only (see the proof of Th. 2.5 iii) to check that ${n_{i}^{\prime}}^{\prime 1 / a}=o\left(a_{n_{i}^{\prime}}\right)$. This follows from $P\left(|X|>a_{n_{i}^{\prime}}\right) \sim 1 / n_{i}^{\prime}$ and $P\left(|X|>a_{n_{i}^{\prime}}\right) \sim L\left(a_{n_{i}^{\prime}}\right) a_{n_{i}^{\prime}}^{-a}$ which give

$$
\frac{a_{n_{i}^{\prime}}^{a}}{n_{i}^{\prime}} \sim L\left(a_{n_{i}^{\prime}}\right) \underset{i \rightarrow \infty}{\longrightarrow} \infty .
$$

So we found two nondecreasing sequences of integers $\left(n_{i}\right)_{i \geq 1}$ and $\left(n_{i}^{\prime}\right)_{i \geq 1}$ such that

$$
\begin{gathered}
n_{i}^{-1 / a} T_{n_{i}}\left(\gamma_{a}\right) \underset{i \rightarrow \infty}{\stackrel{\mathcal{D}}{\longrightarrow}} V_{a, \sigma, \theta} \quad \text { and } \quad c_{n_{i}}^{-1}\left(T_{n_{i}}\left(\gamma_{a}\right)-\mu_{n_{i}}\right) \underset{i \rightarrow \infty}{\stackrel{\mathcal{D}}{\longrightarrow}} Z, \\
b_{n_{i}^{\prime}}^{-1} T_{n_{i}^{\prime}}\left(\gamma_{a}\right) \underset{i \rightarrow \infty}{\stackrel{\mathcal{D}}{\longrightarrow}} V_{a, \sigma, \theta^{\prime}} \text { and } \quad c_{n_{i}^{\prime}}^{-1}\left(T_{n_{i}^{\prime}}\left(\gamma_{a}\right)-\mu_{n_{i}^{\prime}}\right) \underset{i \rightarrow \infty}{\stackrel{\mathcal{D}}{\longrightarrow}} Z,
\end{gathered}
$$

where $b_{n_{i}^{\prime}}={n_{i}^{\prime}}^{1 / a}$ if $\theta^{\prime}<\infty, b_{n_{i}^{\prime}}=a_{n_{i}^{\prime}}$, the $1-1 / n_{i}^{\prime}$ quantile of $X$ if $\theta^{\prime}=\infty$. Applying twice the convergence of types theorem, see [11, Th.1, 2,p.40-42] or [20, Prop.0.2], we deduce from (3.41) and (3.42) that there exist constants $\alpha>0$ and $\beta \in \mathbb{R}$ such that

$$
V_{a, \sigma, \theta} \stackrel{d}{=} \alpha V_{a, \sigma, \theta^{\prime}}+\beta .
$$

As $P\left(V_{a, \sigma, \theta} \leq \varepsilon\right)>0$ for every $\varepsilon>0$, see Appendix A.3, it is easily seen that necessarily $\beta=0$ in (3.43), so we are left with

$$
V_{a, \sigma, \theta} \stackrel{d}{=} \alpha V_{a, \sigma, \theta^{\prime}} .
$$

Now we check the impossibility of the inequality in (3.40) by examinating the following cases.

- $\theta=0<\theta^{\prime} \leq \infty$. Then (3.44) is impossible because $V_{a, \sigma, 0}$ has a subgaussian tail by (1.8), while $\alpha V_{a, \sigma, \theta^{\prime}}$ has an heavy tail equivalent to $\left(\alpha \theta^{\prime}\right)^{a} x^{-a}$ when $0<\theta^{\prime}<\infty$ by (1.10) or to $\alpha^{a} x^{-a}$ when $\theta^{\prime}=\infty$.

- $0<\theta<\theta^{\prime}<\infty$. Looking again at the tails we see by (1.10) that necessarily $\theta=\alpha \theta^{\prime}$, which reformulates (3.44) as

$$
V_{a, \sigma, \theta} \stackrel{d}{=} \frac{\theta}{\theta^{\prime}} V_{a, \sigma, \theta^{\prime}} .
$$


Noticing that for every $x>0$,

$$
\begin{aligned}
P\left(\frac{\theta}{\theta^{\prime}} V_{a, \sigma, \theta^{\prime}} \leq x\right) & =P\left(\frac{\theta}{\theta^{\prime}} \max \left(\sigma T\left(\gamma_{a}\right), \theta^{\prime} Y_{a}\right) \leq x\right) \\
& =P\left(\sigma \frac{\theta}{\theta^{\prime}} T\left(\gamma_{a}\right) \leq x\right) P\left(\theta Y_{a} \leq x\right)
\end{aligned}
$$

we see by comparison with (1.9) that necessarily,

$$
\frac{\theta}{\theta^{\prime}} T\left(\gamma_{a}\right) \stackrel{d}{=} T\left(\gamma_{a}\right)
$$

It is elementary to check that if a non negative random variable $T$ non degenerated to 0 has the same distribution as $c T$ for some constant $c$ then $c=1$. Therefore when $0<\theta<\theta^{\prime}$, (3.44) is impossible.

- $0<\theta<\infty=\theta^{\prime}$. Then (3.44) is equivalent to

$$
P\left(\sigma T\left(\gamma_{a}\right) \leq x\right) P\left(\theta Y_{a} \leq x\right)=P\left(\alpha Y_{a} \leq x\right), \quad x>0,
$$

which we can rewrite as

$$
P\left(\sigma T\left(\gamma_{a}\right) \leq x\right)=\exp \left(\frac{\theta^{a}-\alpha^{a}}{x^{a}}\right)=: G(x), \quad x>0 .
$$

Now if $\theta>\alpha, G$ is not a distribution function. If $\theta=\alpha, G(x)=1$ for every $x>0$ which is clearly not true for the left-hand side of (3.46). If $\theta<\alpha, G$ is the distribution function of a Fréchet distribution with scale parameter $\left(\alpha^{a}-\theta^{a}\right)^{1 / a}$ hence heavy tailed while the d.f. in the left-hand side of (3.46) is subgaussian, so (3.46) is false. Finally in this third case (3.44) cannot be true.

To conclude we have proved that $\theta=\theta^{\prime}$, i.e. that $L(x)$ has a limit $\tau^{a} \in[0, \infty]$ when $x$ tends to infinity. By [16], [12, Th.5b)] and Th.2.4, $b_{n}^{-1} T_{n}\left(\gamma_{a}\right)$ converges in distribution to $V_{a, \sigma, \tau}$ where $b_{n}$ is defined as in (3.42). By the convergence of types theorem applied to the whole sequence we obtain that $c_{n} \sim A b_{n}$ for some positive constant $A$. This shows that the only possible limits in distribution of $T_{n}\left(\gamma_{a}\right)$ under affine normalisation are the random variables $Z=A V_{a, \sigma, \tau}+B, A>0, B \in \mathbb{R}$.

\section{A Appendix}

\section{A.1 Taylor expansions and Lindeberg method}

The following special Taylor expansion is useful when applied to functions of random variables having moments of order $r \in[2,3)$. We applied it in the proofs of Th. 2.2 and Th. 2.4 to the functions $f=f_{r, \varepsilon}$ constructed by Bentkus, denoted $\varphi$ in [2, Th.2], which are $C^{\infty}$.

Lemma A.1. Let $E$ be a Banach space and $f: E \rightarrow \mathbb{R}$ be a $C^{3}$ map with bounded Fréchet second and third derivatives in the sense that

$$
\left\|f^{(m)}\right\|:=\sup \left\{\left\|f^{(m)}(x)\right\|: x \in E\right\}<\infty, \quad m=2,3,
$$

where $\left\|f^{(m)}(x)\right\|$ is the operator norm of the $m$-linear form $f^{(m)}(x): E^{m} \rightarrow \mathbb{R}$, that is

$$
\left\|f^{(m)}(x)\right\|:=\sup \left\{\left|f^{(m)}(x)\left(h_{1}, \ldots, h_{m}\right)\right|:\left\|h_{i}\right\|_{E} \leq 1, i=1, \ldots, m\right\} .
$$

Then for any $x, h \in E$,

$$
f(x+h)=f(x)+f^{\prime}(x)(h)+\frac{1}{2} f^{\prime \prime}(x)(h, h)+R,
$$

where the remainder term $R=R(f, x, h)$ satisfies for $2<\beta \leq 3$,

$$
|R| \leq 6^{2-\beta}\left\|f^{\prime \prime}\right\|^{3-\beta}\left\|f^{\prime \prime \prime}\right\|^{\beta-2}\|h\|_{E}^{\beta} .
$$


Proof. By Taylor formula at the order 2 with integral remainder,

$$
f(x+h)=f(x)+f^{\prime}(x)(h)+\frac{1}{2} f^{\prime \prime}(x)(h, h)+\int_{0}^{1} \frac{(1-t)^{2}}{2} f^{\prime \prime \prime}(x+t h)(h, h, h) \mathrm{d} t,
$$

whence $R:=f(x+h)-f(x)-f^{\prime}(x)(h)-\frac{1}{2} f^{\prime \prime}(x)(h, h)$ is bounded by

$$
|R| \leq \frac{1}{6}\left\|f^{\prime \prime \prime}\right\|\|h\|_{E}^{3}
$$

The Taylor formula at the order 1 with integral remainder provides another bound for $R$. Indeed

$$
\begin{aligned}
f(x+h) & =f(x)+f^{\prime}(x)(h)+\int_{0}^{1}(1-t) f^{\prime \prime}(x+t h)(h, h) \mathrm{d} t \\
& =f(x)+f^{\prime}(x)(h)+\frac{1}{2} f^{\prime \prime}(x)(h, h)+\int_{0}^{1}(1-t)\left(f^{\prime \prime}(x+t h)-f^{\prime \prime}(x)\right)(h, h) \mathrm{d} t,
\end{aligned}
$$

which leads to

$$
|R| \leq\left\|f^{\prime \prime}\right\|\|h\|_{E}^{2}
$$

The bound (A.3) seems preferable for "small" values of $\|h\|_{E}$, while (A.4) can be privilegied for "large" values of $\|h\|_{E}$. More formally, for an arbitrary parameter $t>0$ to be precised later and $2<\beta \leq 3$, we get the bound

$$
|R| \leq \begin{cases}\frac{1}{6}\left\|f^{\prime \prime \prime}\right\|\|h\|_{E}^{\beta} t^{3-\beta} & \text { if }\|h\|_{E} \leq t, \\ \left\|f^{\prime \prime}\right\|\|h\|_{E}^{\beta} t^{2-\beta} & \text { if }\|h\|_{E}>t .\end{cases}
$$

To unify these two bounds, we remark that for $a, b>0, a t^{3-\beta}=b t^{2-\beta}$ for $t=b / a$. With $a=\frac{1}{6}\left\|f^{\prime \prime \prime}\right\|$ and $b=\left\|f^{\prime \prime}\right\|$, this choice of $t$ gives (A.2).

Before providing the justifications of (3.6) and (3.7), we need to introduce some notations. For $m \geq 1$, we denote by $\mathcal{L}_{m}\left(\mathbb{R}^{k}, \mathbb{R}\right)$ the space of $m$-linear forms on $\left(\mathbb{R}^{k}\right)^{m}$. A norm $\|x\|$ being choosen in $\mathbb{R}^{k}$, we denote by $\|g\|_{m}^{*}$ the corresponding operator norm of $g \in \mathcal{L}_{m}\left(\mathbb{R}^{k}, \mathbb{R}\right)$, that is

$$
\|g\|_{m}^{*}:=\sup \left\{\left|g\left(x_{1}, \ldots, x_{m}\right)\right|: x_{i} \in \mathbb{R}^{k},\left\|x_{i}\right\| \leq 1, i=1, \ldots, m\right\} .
$$

As we work with finite dimensional spaces, Pettis and Bochner integrals coincide and we say that a random element in $\mathbb{R}^{k}$ or in $\mathcal{L}_{m}\left(\mathbb{R}^{k}, \mathbb{R}\right)$ is integrable if its norm is an integrable random variable in the usual sense.

Lemma A.2. Let $f^{\prime}$ be a measurable map from $\mathbb{R}^{k}$ into its dual $\mathcal{L}_{1}\left(\mathbb{R}^{k}, \mathbb{R}\right)$ and $X$ and $Z$ be two independent random vectors in $\mathbb{R}^{k}$. Assume moreover that $X$ and $f^{\prime}(Z)$ are integrable. Then $f^{\prime}(Z)(X)$ is an integrable real random variable and

$$
\mathrm{E}\left(f^{\prime}(Z)(X)\right)=\left(\mathrm{E} f^{\prime}(Z)\right)(\mathrm{E} X) .
$$

According to its order of apparition in (A.5), the expectation symbol E denotes successively the expectation of a real valued random variable, of a random linear form on $\mathbb{R}^{k}$ (or random element in $\mathcal{L}_{1}\left(\mathbb{R}^{k}, \mathbb{R}\right)$ ) and of a random vector in $\mathbb{R}^{k}$.

Proof. Denote by $P_{(Z, X)}, P_{X}$ and $P_{Z}$ the respective distributions of $(Z, X), X$ and $Z$. By independence of $Z$ and $X, P_{(Z, X)}$ is the product measure $P_{Z} \otimes P_{X}$. The real valued random variable $f^{\prime}(Z)(X)$ is integrable since

$$
\mathrm{E}\left|f^{\prime}(Z)(X)\right| \leq \mathrm{E}\left(\left\|f^{\prime}(Z)\right\|_{1}^{*}\|X\|\right)=\mathrm{E}\left\|f^{\prime}(Z)\right\|_{1}^{*} \mathrm{E}\|X\|<\infty,
$$


by independence and integrability of $X$ and $f^{\prime}(Z)$. This legitimates the use of Fubini theorem to obtain

$$
\begin{aligned}
\mathrm{E}\left(f^{\prime}(Z)(X)\right)=\int_{\mathbb{R}^{k} \times \mathbb{R}^{k}} f^{\prime}(z)(x) \mathrm{d} P_{(Z, X)}(z, x) & =\int_{\mathbb{R}^{k} \times \mathbb{R}^{k}} f^{\prime}(z)(x) \mathrm{d} P_{Z} \otimes P_{X}(z, x) \\
& =\int_{\mathbb{R}^{k}} \int_{\mathbb{R}^{k}} f^{\prime}(z)(x) \mathrm{d} P_{X}(x) \mathrm{d} P_{Z}(z) \\
& =\int_{\mathbb{R}^{k}} \mathrm{E}\left(f^{\prime}(z)(X)\right) \mathrm{d} P_{Z}(z) \\
& =\int_{\mathbb{R}^{k}} f^{\prime}(z)(\mathrm{E} X) \mathrm{d} P_{Z}(z) \\
& =\left(\mathrm{E} f^{\prime}(Z)\right)(\mathrm{E} X),
\end{aligned}
$$

recalling that if $g=f^{\prime}(z)$ is a linear form on $\mathbb{R}^{k}$ and $\mathrm{E}\|X\|<\infty, g(X)$ is integrable and $\mathrm{E} g(X)=g(\mathrm{E} X)$. So (A.5) is established.

Lemma A.3. Let $f^{\prime \prime}$ be a measurable map from $\mathbb{R}^{k}$ into $\mathcal{L}_{2}\left(\mathbb{R}^{k}, \mathbb{R}\right)$ and $X, Y$ and $Z$ are random vectors in $\mathbb{R}^{k}$ such that

a) $X$ and $Y$ are square integrable ( $\left.\mathrm{E}\|X\|^{2}, \mathrm{E}\|Y\|^{2}<\infty\right)$, $\mathrm{E} X=\mathrm{E} Y=0$;

b) $X$ and $Y$ have the same covariance matrix;

c) $X$ and $Z$ are independent, $Y$ and $Z$ are independent;

d) $f^{\prime \prime}(Z)$ is integrable $\left(\mathrm{E}\left\|f^{\prime \prime}(Z)\right\|_{2}^{*}<\infty\right)$.

Then

$$
\mathrm{E}\left(f^{\prime \prime}(Z)(X, X)\right)=\mathrm{E}\left(f^{\prime \prime}(Z)(Y, Y)\right) \text {. }
$$

Proof. As $f^{\prime \prime}(z)$ is a bilinear form on $\mathbb{R}^{k}$ for each $z \in \mathbb{R}^{k}$, it admits the representation

$$
f^{\prime \prime}(z)(x, y)=\sum_{i, j=1}^{k} a_{i, j}(z) x_{i} y_{j}, \quad x=\left(x_{1}, \ldots, x_{k}\right), y=\left(y_{1}, \ldots, y_{k}\right)
$$

and the integrability of $f^{\prime \prime}(Z)$ implies the integrability of the $k^{2}$ random variables $a_{i, j}(Z)$ because $a_{i, j}(Z)=f^{\prime \prime}(Z)\left(e_{i}, e_{j}\right)$ where $e_{1}, \ldots, e_{k}$ denotes the canonical basis of $\mathbb{R}^{k}$. Writing $X=\left(X_{1}, \ldots, X_{k}\right)$ and $Y=\left(Y_{1}, \ldots, Y_{k}\right)$, we see that

$$
\mathrm{E}\left(f^{\prime \prime}(Z)(X, X)\right)=\mathrm{E}\left(\sum_{i, j=1}^{k} a_{i, j}(Z) X_{i} X_{j}\right)=\sum_{i, j=1}^{k}\left(\mathrm{E} a_{i, j}(Z)\right)\left(\mathrm{E} X_{i} X_{j}\right)
$$

where the last equality uses the square integrability of $X$ which gives the integrability of $X_{i} X_{j}$, the integrability of $a_{i, j}(Z)$ and the independence of $X$ and $Z$. Obviously the same equality holds substituting $X$ by $Y$ and we conclude by b).

\section{A.2 On the use of invariance principles}

Proof of (2.2). We introduce first some notations.

$$
\begin{aligned}
D_{\theta} & :=\left\{(s, u) \in[0,1]^{2} ; 0 \leq s<s+u \leq 1, \theta<u \leq 1\right\}, \\
\overline{D_{\theta}} & :=\left\{(s, u) \in[0,1]^{2} ; 0 \leq s<s+u \leq 1, \theta \leq u \leq 1\right\}, \\
D_{n, \theta} & :=\left\{\left(\frac{k}{n}, \frac{\ell}{n}\right) \in D_{\theta} ; k, \ell \in \mathbb{N}\right\}, \quad n \geq 1 .
\end{aligned}
$$


For every $x \in \mathrm{C}[0,1]$, we set

$$
\tilde{x}(s, u):=\frac{|x(s+u)-x(s)|}{u^{\gamma_{a}}}, \quad(s, u) \in \overline{D_{\theta}}
$$

and define the functionals $g$ and $g_{n}, \mathrm{C}[0,1] \rightarrow \mathbb{R}_{+}$by

$$
g(x):=\sup _{(s, u) \in D_{\theta}} \tilde{x}(s, u), \quad g_{n}(x):=\sup _{(s, u) \in D_{n, \theta}} \tilde{x}(s, u) .
$$

We note that $g$ is Lipschitzian hence continuous on $\mathrm{C}[0,1]$ because it satisfies the inequalities $g(x+y) \leq g(x)+g(y)$, and $0 \leq g(x) \leq 2 \theta^{-\gamma_{a}}\|x\|_{\infty}, x, y \in \mathrm{C}[0,1]$.

Finally we denote by $\xi_{n}$ the Donsker Prohorov polygonal partial sums processes, that is the random polygonal line $\left\{\xi_{n}(t), t \in[0,1]\right\}$ with vertices the points $\left(k / n, S_{k}\right)$, with $S_{0}:=0$. The weak invariance principle in $\mathrm{C}[0,1]$ states that $n^{-1 / 2} \sigma^{-1} \xi_{n}$ converges in distribution to the standard Brownian motion $W$ in the space $\mathrm{C}[0,1]$. By continuous mapping, this implies

$$
g\left(n^{-1 / 2} \sigma^{-1} \xi_{n}\right) \underset{n \rightarrow \infty}{\stackrel{\mathcal{D}}{\longrightarrow}} g(W)=T^{(\theta)}(\gamma)=\max _{\theta<|t-s| \leq 1} \frac{|W(t)-W(s)|}{|t-s|^{\gamma_{a}}} .
$$

Recalling that $\gamma_{a}=1 / 2-1 / a$, we notice that

$$
n^{-1 / a} \sigma^{-1} T_{n}^{n \theta, n}=g_{n}\left(n^{-1 / 2} \sigma^{-1} \xi_{n}\right),
$$

so the weak convergence of $n^{-1 / a} \sigma^{-1} T_{n}^{n \theta, n}$ to $T^{(\theta)}(\gamma)$ will follow from (A.7) if we prove that $g\left(n^{-1 / 2} \sigma^{-1} \xi_{n}\right)-g_{n}\left(n^{-1 / 2} \sigma^{-1} \xi_{n}\right)$ converges to zero in probability.

In what follows, it is convenient to endow $[0,1]^{2}$ with the distance

$$
d\left((s, u),\left(s^{\prime}, u^{\prime}\right)\right)=: \max \left(\left|s-s^{\prime}\right|,\left|u-u^{\prime}\right|\right) .
$$

By continuity of $\tilde{x}$ on $\overline{D_{\theta}}$ and density of $D_{\theta}$ in $\overline{D_{\theta}}, \sup _{D_{\theta}} \tilde{x}=\sup _{\overline{D_{\theta}}} \tilde{x}$ and by compacity of $\overline{D_{\theta}}$ this supremum is reached at some $\left(s_{0}, u_{0}\right) \in \overline{D_{\theta}}$. Hence

$$
g(x)=\tilde{x}\left(s_{0}, u_{0}\right)
$$

It is easily checked that for the distance $d, D_{n, \theta}$ is a $1 / n$ net in $\overline{D_{\theta}}$ in the sense that for any point $(s, u) \in \overline{D_{\theta}}$ there is a point of $D_{n, \theta}$ at a $d$ distance at most $1 / n$ of $(s, u)$. So we can construct a sequence $\left(\left(s_{n}, u_{n}\right)\right)_{n \geq 1}$ such that

$$
\left(s_{n}, u_{n}\right) \in D_{n, \theta} \quad \text { and } \quad d\left(\left(s_{n}, u_{n}\right),\left(s_{0}, u_{0}\right)\right) \leq \frac{1}{n}, \quad n \geq 1 .
$$

Denoting by $\omega(x, \delta):=\sup \{|x(t)-x(s)| ;|t-s| \leq \delta, s, t \in[0,1]\}$, the modulus of uniform continuity of $x$, we get the following estimates valid for any $x \in \mathrm{C}[0,1]$.

$$
\begin{aligned}
\tilde{x}\left(s_{0}, u_{0}\right) & \leq \frac{1}{u_{0}^{\gamma_{a}}}\left(\left|x\left(s_{n}+u_{n}\right)-x\left(s_{n}\right)\right|+2 \omega\left(x, \frac{2}{n}\right)\right) \\
& \leq \frac{\left|x\left(s_{n}+u_{n}\right)-x\left(s_{n}\right)\right|}{u_{0}^{\gamma_{a}}}+2 \theta^{-\gamma_{a}} \omega\left(x, \frac{2}{n}\right) \\
& =\left(\frac{u_{n}}{u_{0}}\right)^{\gamma_{a}} \frac{\left|x\left(s_{n}+u_{n}\right)-x\left(s_{n}\right)\right|}{u_{n}^{\gamma_{a}}}+2 \theta^{-\gamma_{a}} \omega\left(x, \frac{2}{n}\right) \\
& \leq\left(1+\frac{1}{n u_{0}}\right)^{\gamma_{a}} g_{n}(x)+2 \theta^{-\gamma_{a}} \omega\left(x, \frac{2}{n}\right) \\
& \leq\left(1+\frac{1}{n \theta}\right)^{\gamma_{a}} g_{n}(x)+2 \theta^{-\gamma_{a}} \omega\left(x, \frac{2}{n}\right)
\end{aligned}
$$


Applied with any path of the random element $n^{-1 / 2} \sigma^{-1} \xi_{n}$ in $\mathrm{C}[0,1]$ and recalling that $g_{n}(x) \leq g(x)$, this gives

$$
\begin{aligned}
0 \leq g\left(n^{-1 / 2} \sigma^{-1} \xi_{n}\right)-g_{n}\left(n^{-1 / 2} \sigma^{-1} \xi_{n}\right) \leq\left(\left(1+\frac{1}{n \theta}\right)^{\gamma}-1\right) & g\left(n^{-1 / 2} \sigma^{-1} \xi_{n}\right) \\
& +2 \theta^{-\gamma_{a}} \omega\left(n^{-1 / 2} \sigma^{-1} \xi_{n}, \frac{2}{n}\right)
\end{aligned}
$$

As the sequence $\left(n^{-1 / 2} \sigma^{-1} \xi_{n}\right)_{n \geq 1}$ is tight on $\mathrm{C}[0,1]$, its image by the continuous functional $g$ is tight on $\mathbb{R}_{+}$, that is $g\left(n^{-1 / 2} \sigma^{-1} \xi_{n}\right)$ is bounded in probability. Moreover the tightness of $\left(n^{-1 / 2} \sigma^{-1} \xi_{n}\right)_{n \geq 1}$ implies the convergence in probability to 0 of $\sup _{n \geq 1} \omega\left(n^{-1 / 2} \sigma^{-1} \xi_{n}, \delta\right)$ as $\delta$ tends to 0 . This implies that $\omega\left(n^{-1 / 2} \sigma^{-1} \xi_{n}, 2 / n\right)$ tends to 0 in probability as $n$ tends to infinity. All this gives

$$
\left.g_{n}\left(n^{-1 / 2} \sigma^{-1} \xi_{n}\right)\right)=g\left(n^{-1 / 2} \sigma^{-1} \xi_{n}\right)+o_{P}(1) .
$$

Recalling (A.7) and (A.8), this proves the convergence in distribution of $n^{-1 / a} \sigma^{-1} T_{n}^{\theta n, n}$ to $T^{(\theta)}\left(\gamma_{a}\right)$.

Proof of (3.10). We use the Hölderian functional central limit theorem (or Hölderian weak invariance principle) in the space $\mathcal{H}_{\gamma}^{o}[0,1]$ which is defined for any $0<\gamma<1$ as follows. We denote by $\mathcal{H}_{\gamma}^{o}[0,1]$ the space of continuous functions $x$ on $[0,1]$ such that

$$
\omega_{\gamma}(x, \delta):=\sup _{\substack{0<t-s \leq \delta \\ s, t \in[0,1]}} \frac{|x(t)-x(s)|}{(t-s)^{\gamma}} \underset{\delta \rightarrow 0}{\longrightarrow} 0 .
$$

Endowed with the norm $\|x\|_{\gamma}:=|x(0)|+\omega_{\gamma}(x, 1), \mathcal{H}_{\gamma}^{o}[0,1]$ is a separable Banach space.

Let $\left(Y_{k}\right)_{k \geq 1}$ be the Gaussian i.i.d. sequence introduced in the proof of Th.2.2. Puting $\widehat{S_{j}}:=\sum_{i=1}^{j} Y_{i}$ with the convention $\widehat{S_{0}}:=0$, we denote by $\widehat{\xi_{n}}$ the associated polygonal partial sums process, that is the random polygonal line $\left\{\widehat{\xi_{n}}(t), t \in[0,1]\right\}$ with vertices the points $\left(k / n, \widehat{S_{k}}\right), k=0,1, \ldots, n$. As $Y_{1}$ has finite moments of all orders, it follows from the functional central limit theorem proved in [16] that $n^{-1 / 2} \sigma^{-1} \widehat{\xi_{n}}$ converges in distribution in $\mathcal{H}_{\gamma}^{o}[0,1]$ to the standard Brownian motion $W$ for every $0<\gamma<1 / 2$. In particular it converges in $\mathcal{H}_{\gamma_{a}}^{o}[0,1]$. It seems worth noticing here that $n^{-1 / 2} \sigma^{-1} \xi_{n}$, the polygonal partial sums process built on the sequence $\left(X_{k}\right)_{k \geq 1}$, does not converge in $\mathcal{H}_{\gamma_{a}}^{o}[0,1]$. Indeed this convergence requires that $\lim _{t \rightarrow \infty} t^{a} P\left(\left|X_{1}\right|>t\right)=0$, while in the setting of Th. 2.2, this limit is $\tau^{a}>0$.

As the $\gamma$-Hölder norm of a polygonal line is reached at two vertices, see e.g. Lemma 3 in [14], we obtain by continuous mapping,

$$
\left\|n^{-1 / 2} \sigma^{-1} \widehat{\xi_{n}}\right\|_{\gamma_{a}}=n^{-1 / a} \sigma^{-1} \widehat{T}_{n}^{0, n}\left(\gamma_{a}\right) \underset{n \rightarrow \infty}{\stackrel{\mathcal{D}}{\longrightarrow}} T\left(\gamma_{a}\right) \text {. }
$$

So to prove (3.10), it suffices to prove that $n^{-1 / a} \sigma^{-1}\left(\widehat{T}_{n}^{0, n}\left(\gamma_{a}\right)-\widehat{T}_{n}^{d_{n}, n}\left(\gamma_{a}\right)\right)$ converges to 0 in probability if $d_{n} / n$ tends to 0 . To this aim we remark that

$$
\begin{aligned}
0 \leq n^{-1 / a} \sigma^{-1}\left(\widehat{T}_{n}^{0, n}\left(\gamma_{a}\right)-\widehat{T}_{n}^{d_{n}, n}\left(\gamma_{a}\right)\right) & \leq n^{-1 / a} \sigma^{-1} \max _{\substack{0<\ell \leq d_{n} \\
0 \leq k \leq n-\ell}} \frac{\left|\widehat{S}_{k+\ell}-\widehat{S_{k}}\right|}{\left(\frac{\ell}{n}\right)^{\gamma_{a}}} \\
& \leq \omega_{\gamma_{a}}\left(n^{-1 / a} \sigma^{-1} \widehat{\xi}_{n}, \frac{d_{n}}{n}\right) .
\end{aligned}
$$

By Th.13 b) in [23], the tightness in $\mathcal{H}_{\gamma_{a}}^{o}[0,1]$ of $\left(n^{-1 / a} \sigma^{-1} \widehat{\xi}_{n}\right)_{n \geq 1}$, which results from its convergence in distribution in this space, implies for every positive $\varepsilon$ that

$$
\lim _{\delta \rightarrow 0} \sup _{n \geq 1} P\left(\omega_{\gamma_{a}}\left(n^{-1 / a} \sigma^{-1} \widehat{\xi}_{n}, \delta\right)>\varepsilon\right)=0
$$


This in turn, implies when $d_{n} / n \rightarrow 0$, that

$$
\omega_{\gamma_{a}}\left(n^{-1 / a} \sigma^{-1} \widehat{\xi}_{n}, \frac{d_{n}}{n}\right) \underset{n \rightarrow \infty}{\stackrel{\mathrm{P}}{\longrightarrow}} 0
$$

which accounting (A.9) and (A.10), completes the proof.

Theorem A.4. Let $\left(X_{k}\right)_{k \geq 1}$ be i.i.d. with

$$
P\left(\left|X_{1}\right|>x\right)=x^{-a} L(x), \quad x>0,
$$

with $a>2$ and $L$ slowly varying, $\mathrm{E} X_{1}=0, \sigma^{2}=\mathrm{E} X_{1}^{2}$. Let $\xi_{n}$ be the polygonal process built on the partial sums of $\left(X_{k}\right)_{k \geq 1}$. Assume that for some increasing sequence of integers $\left(n_{i}\right)_{i \geq 1}$,

$$
L\left(n_{i}^{1 / a}\right) \underset{i \rightarrow \infty}{\longrightarrow} 0 .
$$

Then $n_{i}^{-1 / 2} \xi_{n_{i}}$ converges weakly to $\sigma W$ in the Hölder space $\mathcal{H}_{\gamma_{a}}^{o}$, where $\gamma_{a}=1 / 2-1 / a$.

Proof. We refer to [16] where the Lamperti invariance principle is proved for the whole sequence $n^{-1 / 2} \xi_{n}$ under the assumption $\lim _{x \rightarrow \infty} x^{a} P\left(\left|X_{1}\right|>x\right)=0$, that is in our notations $\lim _{x \rightarrow \infty} L(x)=0$.

The convergence of finite dimensional distributions follows from the assumption $a>2$ and does not involve $L$. Before looking at the tightness, we note that

$$
\forall t>0, \quad n_{i} P\left(\left|X_{1}\right|>t n_{i}^{1 / a}\right) \underset{i \rightarrow \infty}{\longrightarrow} 0 .
$$

Indeed, puting $x=t n_{i}^{1 / a}$ in (A.11) gives $n_{i} P\left(\left|X_{1}\right|>t n_{i}^{1 / a}\right)=t^{-a} L\left(t n_{i}^{1 / a}\right)$ and as $L$ is slowly varying, $L\left(t n_{i}^{1 / a}\right) \sim L\left(n_{i}^{1 / a}\right)$ which tends to zero by assumption (A.12).

Now for the tightness, following step by step the proof exposed in [16], with the same notations, we obtain first

$$
P_{2}\left(n_{i}, \varepsilon\right) \leq n_{i} P\left(\left|X_{1}\right| \geq \frac{\varepsilon}{2} n_{i}^{1 / a}\right) .
$$

This upper bound tends to 0 by (A.13). The same works for

$$
P_{1,2}\left(J, n_{i}, \varepsilon\right) \leq n_{i} P\left(\left|X_{1}\right| \geq \frac{\varepsilon}{6} n_{i}^{1 / a}\right) .
$$

The hard part of the proof is the treatment of $P_{1,1}\left(J, n_{i}, \varepsilon\right)$ where the $X_{k}$ are truncated at the level $\delta n_{i}^{1 / a}$. Everything around the use of Rosenthal inequality works replacing $n$ by $n_{i}$, except maybe the control of the moments of the truncated variables $\widetilde{X}_{k}=$ $X_{k} \mathbb{1}\left\{\left|X_{k}\right| \leq \delta n_{i}^{1 / a}\right\}$ which can be achieved by the following adaptations.

Since $\mathrm{E} X_{1}=0, \mathrm{E} \tilde{X}_{1}=-\mathrm{E} X_{1} \mathbb{1}\left\{\left|X_{1}\right|>\delta t n_{i}^{1 / a}\right\}$. By a Fubini argument,

$$
\mathrm{E}\left(\left|X_{1}\right| \mathbb{1}\left\{\left|X_{1}\right|>\delta n_{i}^{1 / a}\right\}\right)=\delta n_{i}^{1 / a} P\left(\left|X_{1}\right|>\delta n_{i}^{1 / a}\right)+\int_{\delta n_{i}^{1 / a}}^{\infty} P\left(\left|X_{1}\right|>s\right) \mathrm{d} s .
$$

The first term in the right-hand side above tends to 0 by (A.13). To treat the second term, we cannot use here $\sup _{s>0} s^{a} P\left(\left|X_{1}\right|>s\right)<\infty$ like in [16], but we can exploit the slow variation of $L$ (which was not supposed in [16]). Indeed by Karamata A.4-Th.A.13,

$$
\int_{\delta n_{i}^{1 / a}}^{\infty} s^{-a} L(s) \mathrm{d} s \sim \frac{1}{a-1} \delta n_{i}^{1 / a}\left(\delta n_{i}^{1 / a}\right)^{-a} L\left(\delta n_{i}^{1 / a}\right) \sim \frac{1}{a-1} \delta^{1-a} n_{i}^{1 / a-1} L\left(n_{i}^{1 / a}\right) .
$$

Since $L\left(n_{i}^{1 / a}\right) \rightarrow 0$, there is a constant $c$ such that $\mathrm{E}\left|\widetilde{X_{k}}\right| \leq c \delta^{-a+1} n_{i}^{-1+1 / a}$, like in [16]. 
The other truncated moment we have to take care of is $\mathrm{E}\left|{\widetilde{X_{k}}}^{q}\right|$, for $q>a$. We use again Karamata theorem and slow variation of $L$ as follows.

$$
\begin{aligned}
\mathrm{E}\left|\widetilde{X}_{k}^{q}\right| \leq \int_{0}^{\delta n_{i}^{1 / a}} u^{q-1} P\left(\left|X_{1}\right|>u\right) \mathrm{d} u & =\int_{0}^{\delta n_{i}^{1 / a}} u^{q-a-1} L(u) \mathrm{d} u \\
& \sim \frac{1}{q-a} \delta n_{i}^{1 / a}\left(\delta n_{i}^{1 / a}\right)^{q-a-1} L\left(\delta n_{i}^{1 / a}\right) \\
& \sim \frac{1}{q-a} \delta^{q-a} n_{i}^{q / a-1} L\left(n_{i}^{1 / a}\right)
\end{aligned}
$$

As $L\left(n_{i}^{1 / a}\right)$ tends to zero, one can again find a constant $c$ such that $\mathrm{E}\left|\widetilde{X}_{k}^{q}\right| \leq c \delta^{q-a} n_{i}^{q / a-1}$ like in [16].

There is no other modification to do in the proof presented in [16].

\section{A.3 Continuity of the distribution function of $T(\gamma)$}

Here we justify the continuity of the distribution function $F(r)=P\left(\|W\|_{\gamma} \leq r\right)$ for $0<\gamma<1 / 2$.

Let us check first the continuity at $r=0$. Since obviously $F(r)=0$ for $r<0$ and $F$ is right continuous everywhere, this continuity is equivalent to $F(0)=0$. As $\omega_{\gamma}(x, 1)=0$ implies that $x(1)=x(0)$,

$$
F(0)=P\left(\|W\|_{\gamma}=0\right) \leq P(W(1)-W(0)=0)=0,
$$

since $W(1)-W(0)$ is standard normal distributed.

Next we claim that $F(r)>0$ for every $r>0$. To see this, it may be convenient to use the equivalent Ciesielski's sequential norm $\|W\|_{\gamma}^{\text {seq }}$ built on the weighted dyadic second differences of $W$, see e.g. [18]. In particular, for $0<\gamma<1 / 2$, there is a positive constant $c_{\gamma}$ such that $\|W\|_{\gamma}^{\text {seq }} \geq c_{\gamma}\|W\|_{\gamma}$. By [18, Th.4.1], the distribution function of $\|W\|_{\gamma}^{\text {seq }}$ is

$$
G(t):=\mathrm{P}\left(\|W\|_{\gamma}^{\mathrm{seq}} \leq t\right)=\operatorname{erf}\left(2^{-1 / 2} t\right) \prod_{j=1}^{\infty}\left\{\operatorname{erf}\left(2^{j(1 / 2-\gamma)} t\right)\right\}^{2^{j-1}}, \quad t>0
$$

where erf $t:=\frac{2}{\sqrt{\pi}} \int_{0}^{t} \exp \left(-s^{2}\right) \mathrm{d} s$ and the support of the distribution of $\|W\|_{\gamma}^{\text {seq }}$ is $[0, \infty)$ because $\lim _{j \rightarrow \infty} j^{1 / 2} 2^{j(\gamma-1 / 2)}=0$. In particular $G(t)>0$ for every $t>0$. Applying this with $t=c_{\gamma} r, r>0$, we obtain

$$
0<P\left(\|W\|_{\gamma}^{\text {seq }} \leq c_{\gamma} r\right) \leq P\left(\|W\|_{\gamma} \leq r\right),
$$

that is $F(r)>0$ for every $r>0$.

To establish the continuity of $F$ on $(0, \infty)$, we use the following Ehrhard's theorem.

Theorem A.5 ([7, Th.3.3]). Let $(E, \mu)$ be a Gauss space: $E$ is a locally convex Hausdorff space and $\mu$ a Gaussian Radon measure on $E$; for any $A, B$ convex Borelian subsets of $E$ and every $u \in(0,1)$, it holds

$$
\left(\Phi^{-1} \circ \mu\right)(u A+(1-u) B) \geq u\left(\Phi^{-1} \circ \mu\right)(A)+(1-u)\left(\Phi^{-1} \circ \mu\right)(B),
$$

where $\Phi^{-1}:(0,1) \rightarrow \mathbb{R}$ is the inverse map of $\Phi: t \mapsto \Phi(t):=\frac{1}{\sqrt{2 \pi}} \int_{-\infty}^{t} \exp \left(-s^{2} / 2\right) \mathrm{d} s$.

Applying (A.15) to $A$ and $B$ closed balls with center 0 in $\mathcal{H}_{\gamma}^{o}[0,1]$ gives the concavity of the function $\Phi^{-1}(F(r))$ on $\left(F^{\leftarrow}(0), \infty\right)$ where $F^{\leftarrow}(0):=\inf \{r \in \mathbb{R}: F(r)>0\}$. As seen above, $F^{\leftarrow}(0)=0$, so this concavity implies the continuity of $\Phi^{-1} \circ F$ hence also of $F$ on the open interval $(0, \infty)$. As moreover $F$ is continuous at 0 , the continuity of $F$ on $\mathbb{R}$ is established. 


\section{A.4 Slow variation}

We gathered here some properties of slow variation used in the paper.

Definition A.6. Let $L$ be a positive measurable function, defined in some neighborhood $[A, \infty)$ of infinity and satisfying

$$
\lim _{x \rightarrow \infty} \frac{L(c x)}{L(x)}=1, \quad \forall c>0 .
$$

Then $L$ is said to be slowly varying.

Remark A.7. Every measurable function equivalent to a slowly varying function when $x$ tends to infinity is itself slowly varying on some $[B, \infty)$.

Proposition A.8 (Prop.1.3.6 in [4]). i) If $L$ varies slowly, so does $(L(x))^{a}$ for every $a \in \mathbb{R}$.

ii) If $L$ varies slowly and $a>0$, then $L\left(x^{a}\right)$ varies slowly.

iii) If $L_{1}, L_{2}$, vary slowly, so do $L_{1}+L_{2}, L_{1} L_{2}$ and $\frac{L_{1}}{L_{2}}$.

iv) If $L_{1}, \ldots, L_{k}$, vary slowly and $R\left(x_{1}, \ldots, x_{k}\right)$ is a rational fraction with positive coefficients, $R\left(L_{1}(x), \ldots, L_{k}(x)\right)$ varies slowly.

Theorem A.9 (uniform convergence theorem [4, Th.1.2.1]). If $L$ varies slowly, then for every compact subset $K$ of $(0, \infty)$,

$$
\frac{L(c x)}{L(x)} \underset{x \rightarrow \infty}{\longrightarrow} 1, \quad \text { uniformly in } c \in K
$$

Corollary A.10. i) If $L_{1}$ and $L_{2}$ vary slowly and $\lim _{x \rightarrow \infty} L_{2}(x)=\infty$, then $L_{1} \circ L_{2}$ varies slowly.

ii) If $L$ varies slowly varying and $f$ verifies $\inf _{x \geq B} f(x)>0, \sup _{x \geq B} f(x)<\infty$, then $L(x f(x)) \sim L(x)$ and varies slowly.

Theorem A.11 (representation theorem [4, Th.1.3.1]). The function $L$ is slowly varying if and only if it may be written in the form

$$
L(x)=c(x) \exp \left\{\int_{A}^{x} \frac{\varepsilon(u)}{u} \mathrm{~d} u\right\}, \quad x \geq A,
$$

for some $A>0$, where $c, \varepsilon$ are measurable functions with $c$ positive, $c(x) \rightarrow C \in(0, \infty)$, $\varepsilon(u) / u$ is locally integrable on $[A, \infty)$ and $\varepsilon(x) \rightarrow 0$ as $x \rightarrow \infty$.

Corollary A.12. If $L$ varies slowly, then for every positive real $a, x^{a} L(x)$ is equivalent to a function which increases ultimately to infinity and $x^{-a} L(x)$ equivalent to a function which decreases ultimately to 0.

A positive measurable function $G$ defined on some neighborhood $[A, \infty)(A \geq 0)$ of infinity and satisfying for some real $p$ and every $y>0$,

$$
\frac{G(x y)}{G(x)} \underset{x \rightarrow \infty}{\longrightarrow} y^{p},
$$

is said regularly varying (at infinity) with exponent $p$. In the special case where $p=0, G$ is slowly varying. It is easily seen that each regulary varying function $G$ with exponent $p$ can be writen as $G(x)=x^{p} L(x)$ where $L$ is slowly varying.

Assuming for notational simplicity that $A=0$ and that $G$ is locally bounded and regularly varying with exponent $p$, let us define for $r$ real,

$$
G_{r}(x)=\int_{0}^{x} y^{r} G(y) \mathrm{d} y, \quad G_{r}^{*}(x)=\int_{x}^{\infty} y^{r} G(y) \mathrm{d} y .
$$


Theorem A.13 (Karamata [10, Th.1, p.281]). For $G$ regularly varying with exponent $p$,

i) if $G_{r}^{*}(x)$ is finite (for some and then for every positive $x$ ),

$$
\frac{x^{r+1} G(x)}{G_{r}^{*}(x)} \underset{x \rightarrow \infty}{\longrightarrow} \lambda=-(r+p+1) \geq 0 ;
$$

ii) if $r \geq-p-1$, then

$$
\frac{x^{r+1} G(x)}{G_{r}(x)} \underset{x \rightarrow \infty}{\longrightarrow} \lambda=r+p+1 .
$$

\section{References}

[1] P. Baldi and B. Roynette, Some exact equivalents for the Brownian motion in Hölder norm, Proba. Theory Relat. Fields 93 (1992), 457-484. MR-1183887

[2] V. Bentkus, Smooth approximation of the norm and differentiable functions with bounded support in Banach space $l_{\infty}^{k}$, Lithuanian Math. J. 30 (1990), 489-499. MR-1082475

[3] J. Bertoin, Darling-Erdös theorems for normalized sums of i.i.d. variables close to stable law, Ann. Probab. 26 (1998), no. 2, 832-852. MR-1626527

[4] N. H. Bingham, C. M. Goldie, and J. L. Teugels, Regular variation, Encyclopaedia of Mathematics and its Applications, Cambridge University Press, 1987. MR-1015093

[5] M. Csörgő and P. Révész, Strong approximations in probability and statistics, Academic Press, New York, 1981. MR-0666546

[6] D. A. Darling and P. Erdös, A limit theorem for the maximum of normalized sums of independent random variables, Duke Math. J. (1956), 23143-154. MR-0074712

[7] A. Ehrhard, Symétrisation dans l'espace de Gauss, Math. Scand. 53 (1983), 281-301. MR0745081

[8] U. Einmahl, The Darling-Erdös theorem for sums of i.i.d. random variables, Probab. Th. Rel. Fields 82 (1989), 241-257. MR-0998933

[9] V. R. Fatalov, Large deviations for Gaussian processes in Hölder norm, Izvestiya: Mathematics 67 (2003), no. 5, 1061-1079. MR-2018746

[10] W. Feller, An introduction to probability theory and its applications, second ed., vol. 2, Wiley, 1971. MR-0270403

[11] B. V. Gnedenko and A. N. Kolmogorov, Limit distributions for sums of independent random variables, Addison-Wesley, 1954. MR-0062975

[12] J. Gudan, A. Račkauskas, and Ch. Suquet, Testing mean changes by maximal ratio statistics, To appear in Extremes (2021).

[13] Z. Kabluchko and Y. Wang, Limiting distribution for the maximal standardized increment of a random walk, Stochastic Processes Appl. 124 (2014), 2824-2867. MR-3217426

[14] J. Markevičiūtè, A. Račkauskas, and Ch. Suquet, Functional central limit theorems for sums of nearly nonstationary processes, Lithuanian Math. J. 52 (2012), no. 3, 282-296. MR-3020943

[15] T. Mikosch and A. Račkauskas, The limit distribution of the maximum increment of a random walk with regularly varying jump size distribution, Bernoulli 16 (2010), no. 4, 1016-1038. MR-2759167

[16] A. Račkauskas and Ch. Suquet, Necessary and sufficient condition for the Lamperti invariance principle, Theory of Probability and Mathematical Statistics 68 (2003), 115-124. MR-2000642

[17] A. Račkauskas and Ch. Suquet, Hölder norm test statistics for epidemic change, Journal of Statistical Planning and Inference 126 (2004), no. 2, 495-520. MR-2088755

[18] A. Račkauskas and Ch. Suquet, Computing the distribution of sequential Hölder norms of the Brownian motion, Communications in Statistics - Theory and Methods 45 (2016), no. 15, 4378-4391. MR-3509526

[19] A. Račkauskas and Ch. Suquet, On Bernstein-Kantorovich invariance principle in Hölder spaces and weighted scan statistic, ESAIM: PS 24 (2020), 186-206. MR-4072633 
Maximal weighted increment asymptotic

[20] S. I. Resnick, Extreme values, regular variation, and point processes, Springer, 1987. MR0900810

[21] P. Révész, Random walk in random and non-random environments, World Scientific, Singapore, 1990. MR-1082348

[22] Q. M. Shao, On a conjecture of Révész, Proc. Amer. Math. Soc. 123 (1995), no. 2, 575-582. MR-1231304

[23] Ch. Suquet, Tightness in Schauder decomposable Banach spaces, Amer. Math. Soc. Transl., vol. 193, AMS, 1999, pp. 201-224. MR-1736910 


\section{Electronic Journal of Probability Electronic Communications in Probability}

\section{Advantages of publishing in EJP-ECP}

- Very high standards

- Free for authors, free for readers

- Quick publication (no backlog)

- Secure publication $\left(\mathrm{LOCKSS}^{1}\right)$

- Easy interface (EJMS²)

\section{Economical model of EJP-ECP}

- Non profit, sponsored by $\mathrm{IMS}^{3}, \mathrm{BS}^{4}$, ProjectEuclid ${ }^{5}$

- Purely electronic

\section{Help keep the journal free and vigorous}

- Donate to the IMS open access fund ${ }^{6}$ (click here to donate!)

- Submit your best articles to EJP-ECP

- Choose EJP-ECP over for-profit journals

\footnotetext{
${ }^{1}$ LOCKSS: Lots of Copies Keep Stuff Safe http://www. lockss.org/

${ }^{2}$ EJMS: Electronic Journal Management System http://www.vtex.lt/en/ejms.html

${ }^{3}$ IMS: Institute of Mathematical Statistics http://www.imstat.org/

${ }^{4}$ BS: Bernoulli Society http://www. bernoulli-society.org/

${ }^{5}$ Project Euclid: https://projecteuclid.org/

${ }^{6}$ IMS Open Access Fund: http://www.imstat.org/publications/open.htm
} 